\title{
Intercomparaison de méthodes d'échantillonnage des matières en suspension en milieu fluvial: application à la mesure de la concentration en micropolluants métalliques
}

\author{
Comparison of methods for sampling \\ suspended matter in rivers : application to measurement \\ of particulate metals
}

\author{
Ciffroy P., Vazelle D., Mataix V.(*)
}

Taconnet J., Estèbe A., Thévenot D. $\left.{ }^{* *}\right)$

Bourguignon O., Idlafkih Z., Meybeck M. $\left.{ }^{(* *}\right)$

(*) EDF, Direction des Etudes et Recherches, Département Environnement. 6, quai Watier, 78400 Chatou. France.

(*) Laboratoire de Bioélectrochimie et d'Analyse du Milieu (LABAM). Université Paris XII-Val de Marne. 61, Ave du Général de Gaulle, 94010 Créteil. France.

(***) Laboratoire de Géologie Appliquée, Université P. et M. Curie. 4, Place Jussieu, 75252 Paris Cédex 05. France.

Résumé. - Le but de l'étude décrite dans le présent article est de comparer plusieurs méthodes de prélèvement de matières en suspension (centrifugation, prélèvement séquentiel d'eau et filtration, filtration sur cartouches, trappes immergées, décanteur émergé (dit MIM), afin d'évaluer la représentativité de l'échantillonnage et la faisabilité des techniques testées pour l'évaluation des concentrations en micropolluants métalliques particulaires. A cette fin, deux campagnes d'intercomparaison ont été organisées en aval de la centrale nucléaire de Nogent/Seine, au cours de deux périodes hydrologiques contrastées. La représentativité des diverses techniques mises en jeu a été testée par le suivi de la concentration totale en divers métaux traces ( $\mathrm{Cu}, \mathrm{Zn}, \mathrm{Fe}, \mathrm{Mn})$, par la mesure des spectres granulométriques des échantillons recueillis et par l'évaluation de la spéciation biogéochimique des métaux particulaires.

Mots clés. - Échantillonnage, Matières en suspension, Métaux.

Abstract. - This study aims at comparing several methods of sampling suspended matter to measure particulate metals (centrifuging, sequential sampling and filtration, cartridge filtration, immersed traps, emerged decanter). Two field experiments were undertaken downstream of the Nogent/Seine nuclear power plant, during a flood period 
and a dry summer period. The methods examined were compared on the basis of several criteria : total particulate metal concentrations ( $\mathrm{Cu}, \mathrm{Zn}, \mathrm{Fe}, \mathrm{Mn})$, granulometric spectra of several samples, bio-geochemical speciation of particulate metals.

Key words. - Sampling methods, Suspended matter, Metals.

\section{INTRODUCTION}

Les matières en suspension (MES) constituent l'un des vecteurs majeurs de transport des micropolluants métalliques en rivière. Pour pouvoir évaluer le niveau de contamination d'un cours d'eau, il est donc souvent essentiel de disposer d'une série de mesures relatives à la phase particulaire en suspension : caractérisation physico-chimique des particules (teneur en carbone organique et inorganique, spectre granulométrique,...); concentration totale des micropolluants métalliques associés aux particules; spéciation chimique des micropolluants particulaires, permettant de prévoir leur remobilisation potentielle. Pour pouvoir assurer l'ensemble de ces mesures, il est nécessaire de recueillir d'importantes quantités de matériel particulaire. A cette fin, plusieurs techniques de prélèvement des MES peuvent être mises en œuvre: centrifugation en continu (Duinker et al., 1976 ; Leenaers, 1989), prélèvement séquentiel d'eau et filtration frontale, filtration sur cartouches (Battaglia et al., 1984 ; Ciffroy et al., 1995(1)), filtration tangentielle (Sansone, commu- nication personnelle), trappes immergées (Gardner, 1980), décanteur émergé (Ciffroy et al., 1994). Le choix de la technique utilisée est généralement dicté par des critères de coût et d'autonomie sur le long terme. II conviendrait toutefois d'orienter la sélection de méthodes de prélèvement de MES selon des critères de représentativité plus objectifs : respect du seuil de coupure (arbitrairement fixé à $0,45 \mu \mathrm{m}$ ) et présence négligeable de colloïdes dans l'échantillon; respect du spectre granulométrique; intégrité de la nature des particules; utilisation de matériaux inertes vis à vis des micropolluants à doser (absence d'adsorption ou de relargage de métaux par le système de prélèvement).

Le but de l'étude présentée ici est de comparer plusieurs méthodes de prélèvement de MES, afin d'évaluer la représentativité de l'échantillonnage et la faisabilité des techniques testées pour l'évaluation de concentrations en micropolluants métalliques particulaires. A cette fin, deux campagnes d'intercomparaison ont été organisées en aval de la centrale nucléaire de Nogent/Seine, au cours de deux périodes hydrologiques con- 
trastées. La représentativité des diverses techniques mises en jeu a essentiellement été testée par le suivi de métaux traces, en l'occurrence le cuivre et le zinc, émis par la corrosionérosion des tubes de condenseurs des circuits de refroidissement de la centrale.

\section{CRITĖRES DE REPRÉSENTATIVITÉ ET TECHNIQUES DE PRÉLÈVEMENT}

\subsection{Critères de représentativité}

Plusieurs critères doivent être respectés pour assurer qu'un échantillon de particules soit effectivement représentatif du milieu naturel:

- le seuil de coupure: par définition, sont considérés comme "matières en suspension" l'ensemble des composés dont la taille est supérieure à une limite arbitrairement fixée (généralement, $0,45 \mu \mathrm{m})$. La méthode de prélèvement doit permettre de séparer les composés du milieu selon ce critère de taille. En particulier, il convient d'éviter la présence de colloïdes dans l'échantillon final. Ces derniers, étant en partie constitués de composés dont la taille est proche du seuil de coupure (acides humiques de taille comprise entre $100 \mathrm{kDa}$ et $0,45 \mu \mathrm{m}$ ), peuvent en effet «contaminer» l'échantillon particulaire et provoquer, compte tenu de leur forte affinité pour certains métaux, une surestimation des concentrations métalliques particulaires.

- le spectre granulométrique: les MES présentes dans une rivière en un lieu et un instant donnés sont constituées par un ensemble de particules diverses, distribuées selon un spectre granulométrique spécifique. Or, l'affinité des particules pour les métaux dépend en partie de leur taille : les particules les plus fines présentent en général les concentrations massiques en micropolluants les plus élevées (Ciffroy et al., 1995(2)). Il importe donc que la méthode de prélèvement sélectionnée ne privilégie pas telle fraction granulométrique au détriment de telle autre.

- Ia nature des particules: les MES sont constituées de divers types de particules: argiles, matière organique vivante ou détritique, oxydes et hydroxydes de fer ou de manganèse, précipités de carbonates. Un métal donné se distribue parmi l'ensemble de ces supports particulaires, selon leur abondance respective et l'affinité des interactions métal-support. Aussi est-il essentiel de ne pas discriminer, au cours du prélèvement, l'un des supports particulaires (par exemple par destruction de la matière organique vivante, dissolution de précipités,...) et de ne pas déplacer ainsi le spectre qualitatif des MES.

- l'absence d'échanges de métaux à linterface liquide-solide: au cours du prélèvement, les particules, initialement contenues dans un large volume d'eau, sont regroupées dans un 
volume restreint présentant des caractéristiques physico-chimiques différentes de celle du milieu naturel (teneur en eau, conditions rédox,...). Les équilibres chimiques peuvent donc être modifiés au cours du processus de concentration des MES. L'effet de ces modifications, inhérent à chacune des méthodes de prélèvement employées, doit être estimée par des tests comparatifs. Par ailleurs, au cours de l'échantillonnage, l'eau prélevée entre inévitablement en contact avec certains matériaux du système employé (paroi de la centrifugeuse, filtre,...). Certains de ces matériaux peuvent interagir avec les métaux contenus dans l'eau échantillonnée, ou sont susceptibles de relarguer eux-mêmes des métaux, provoquant ainsi une contamination des MES prélevées. Au cours d'un prélèvement, il importe donc de limiter l'ensemble des échanges «parasites " pouvant se produire aux interfaces liquide-solide.

\subsection{Techniques de prélèvement}

Diverses techniques peuvent être mises en œuvre pour l'échantillonnage de MES en vue de la mesure de la contamination du milieu par des micropolluants métalliques. II importe d'évaluer, a priori, les avantages et les limites de chacune de ces techniques au regard des critères de représentativité précédemment évoqués. En plus de ces critères, le choix d'une méthode de prélèvement est égale- ment dicté par des notions pratiques: coût du prélèvement, autonomie de système, masse et forme des échantillons recueillis, devant être compatibles avec les mesures ultérieures prévues. Dans le présent paragraphe seront décrits brièvement les avantages et les limites de chacune des techniques (les avantages figureront en gras et les limites en italiques); le tableau 1 fournit une vision récapitulative des points évoqués ci-dessous.

- Centrifugation en continu: L'eau de rivière est acheminée par pompage vers un bol tournant à grande vitesse (> $10000 \mathrm{tr} / \mathrm{min}$ ); sous l'effet de la force centrifuge, les particules sont plaquées contre les parois du bol et peuvent être récupérées en fin de manipulation. Cette technique présente a priori de bons rendements d'extraction et permet d'obtenir en quelques heures une quantité importante de matière. Cette dernière se présente sous forme d'un concentrat utilisable pour l'ensemble des mesures classiquement souhaitées. Toutefois, il est impossible d'établir avec précision la limite granulométrique d'extraction des particules; il n'est donc pas exclu que cette technique provoque non seulement le prélèvement de MES, mais aussi de colloïdes. La séparation s'effectuant selon un critère massique, il est possible que les spectres granulométrique et qualitatif des MES recueillies ne soient conformes à ceux du milieu naturel. Par ailleurs, les techniques de centrifugation nécessitent des investissements coûteux et une sur- 


\begin{tabular}{|c|c|c|c|c|c|}
\hline 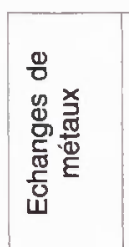 & 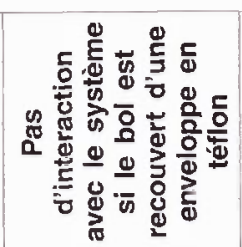 & 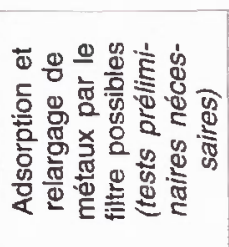 & 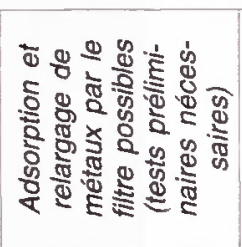 & 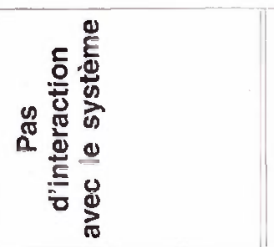 & 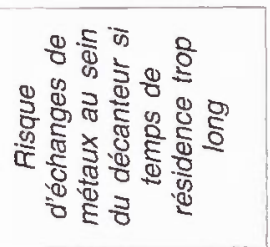 \\
\hline 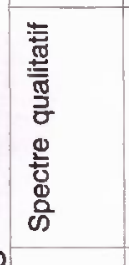 & 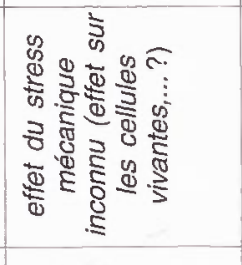 & 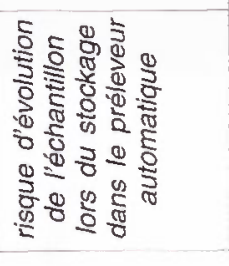 & 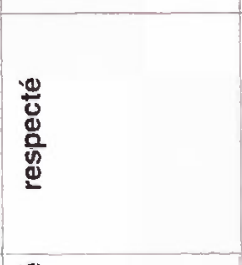 & 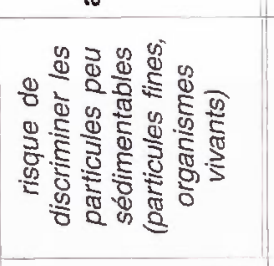 & 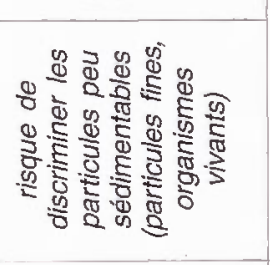 \\
\hline 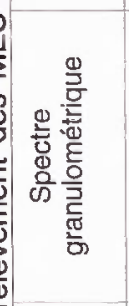 & 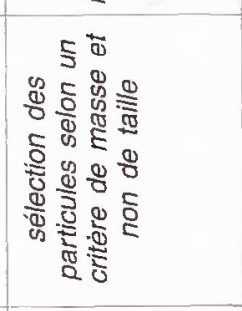 & 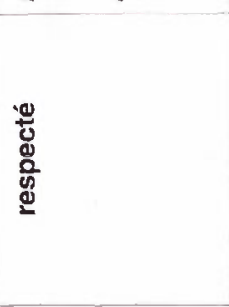 & 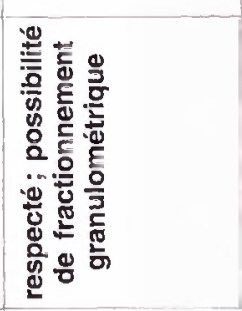 & 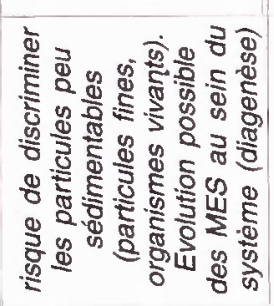 & 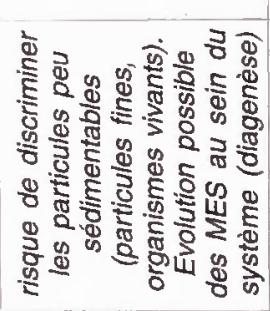 \\
\hline 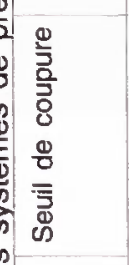 & 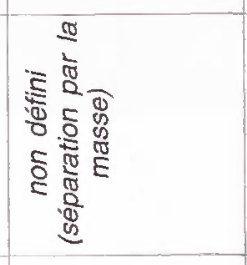 & 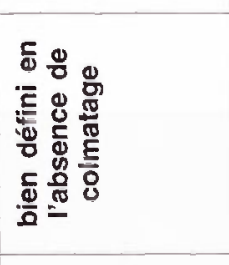 & 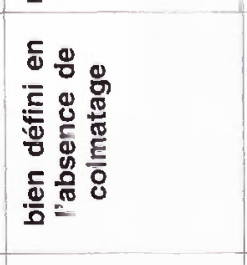 & 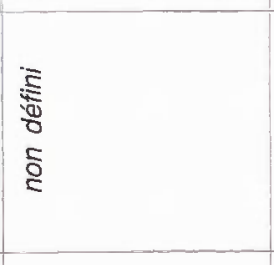 & 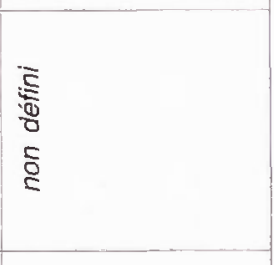 \\
\hline 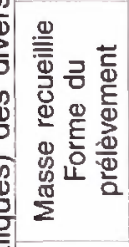 & 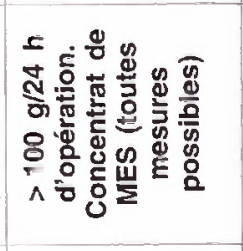 & 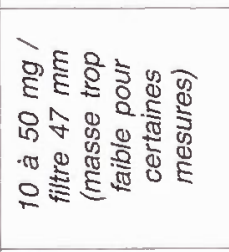 & 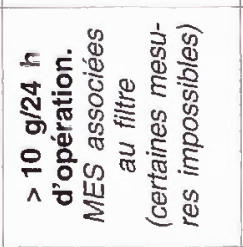 & 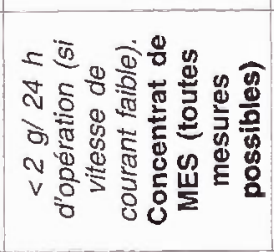 & 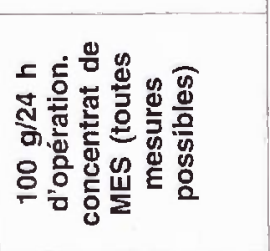 \\
\hline 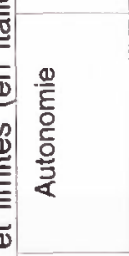 & 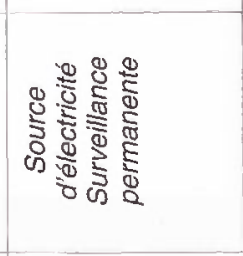 & 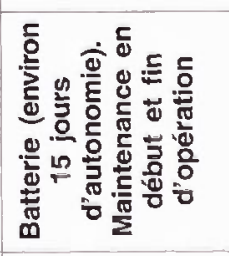 & 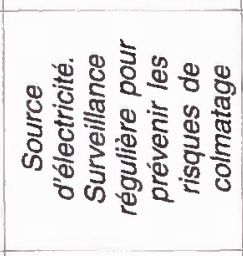 & 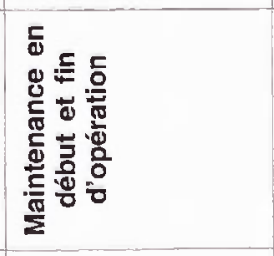 & 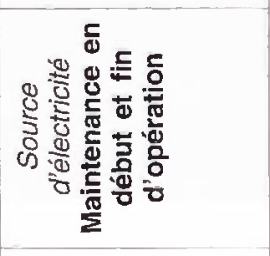 \\
\hline$\overline{\mathrm{o}}$ & 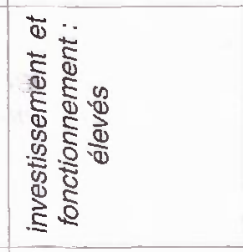 & 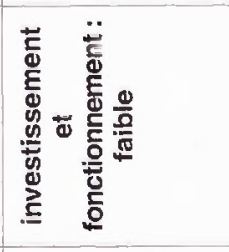 & 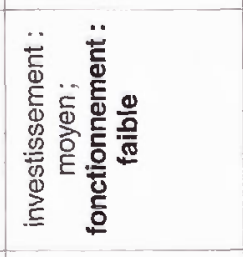 & 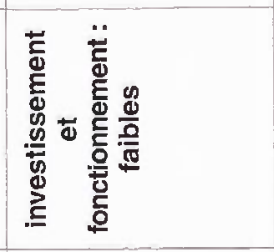 & 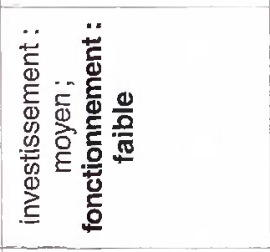 \\
\hline & 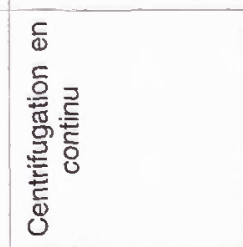 & 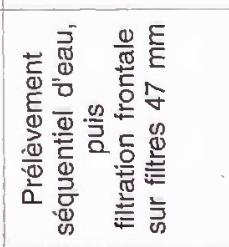 & 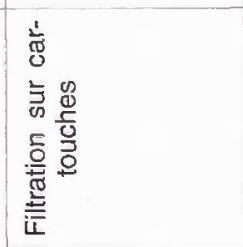 & 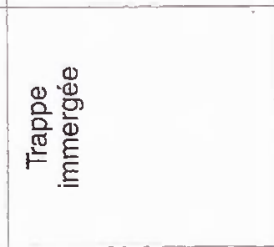 & 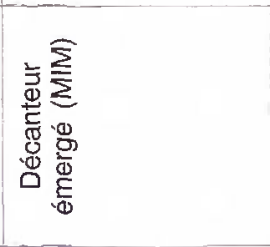 \\
\hline
\end{tabular}


veillance quasi permanente du matériel.

- Prélèvement séquentiel d'eau et filtration: Des échantillons d'eau sont pompés à fréquence régulière par l'intermédiaire d'un préleveur automatique programmable pouvant fonctionner de manière autonome sur batterie (préleveur de type ISCO). Ces échantillons sont ensuite stockés dans des bidons placés à l'obscurité. Les préleveurs classiques présentent généralement une capacité de stockage limitée (de l'ordre de 20 litres). L'eau ainsi recueillie peut ultérieurement être filtrée; compte tenu des volumes à traiter, on utilise généralement des filtres de taille réduite (diamètre des filtres classiquement em- ployés $=47 \mathrm{~mm}$ ). Bien que la technique permette effectivement de recueillir des MES en respectant le seuil de coupure et les spectres granulométrique et qualitatif (à condition qu'il n'y ait pas colmatage des filtres), la masse finale des MES collectées est très réduite et nombre de mesures s'avèrent par conséquent impossibles. Certaines précautions doivent par ailleurs être prises pour éviter le relargage de plusieurs métaux par certains types de filtres.

- Filtration sur cartouche(s) (figure 1) : l'eau de rivière est injectée en tête d'une rampe de filtration par le biais d'une pompe péristaltique; elle remplit un carter en plastique dans lequel est placée une cartouche fil-

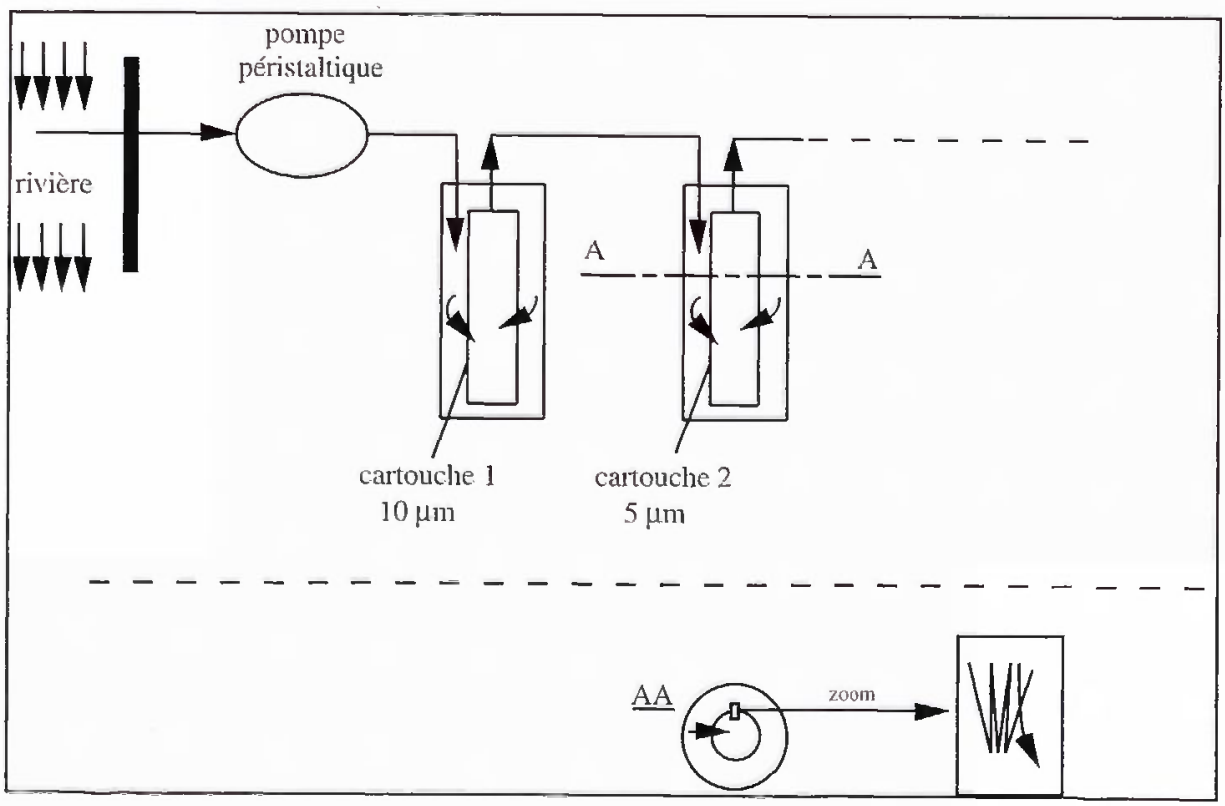

Fig. 1. - Schéma de principe du système de filtration sur cartouches (exemple du système EDF) Fig. 1. - Scheme of cartridges filtration system (example: system used by EDF) 
trante de seuil de coupure défini et de grande surface $\left(>0,5 \mathrm{~m}^{2}\right)$; l'eau est alors filtrée de l'extérieur de la cartouche vers l'intérieur, puis injectée dans la cartouche suivante, et ainsi de suite jusqu'à la sortie. La grande surface des filtres employés permet de traiter de grandes quantités d'eau en minimisant les risques de colmatage, et donc de recueillir d'importantes quantités de matière en respectant les seuils de coupure et les spectres granulométrique et qualitatif. L'utilisation de plusieurs filtres en série permet par ailleurs de séparer plusieurs fractions granulométriques des MES, sur lesquelles des mesures de contamination métallique peuvent être respectivement réalisées. Les MES collectées sont toutefois associées au filtre, ce qui empêche d'envisager certaines mesures (teneur en matière organique des MES, spéciation des métaux particulaires,...). Aucune étude n'a par ailleurs permis à ce jour d'évaluer les problèmes de contamination de l'échantillon à la suite de l'adsorption ou du relargage de métaux par le filtre lui-même. Signalons en outre que cette méthode nécessite une source d'électricité et exige une surveillance régulière pour prévenir les risques de colmatage des filtres, pouvant intervenir lors d'un fonctionnement sur le long terme du système.

- Trappe immergée (figure 2) : une trappe à MES est constituée par un récipient ouvert vers le haut, à l'intérieur duquel est placé un matériau en

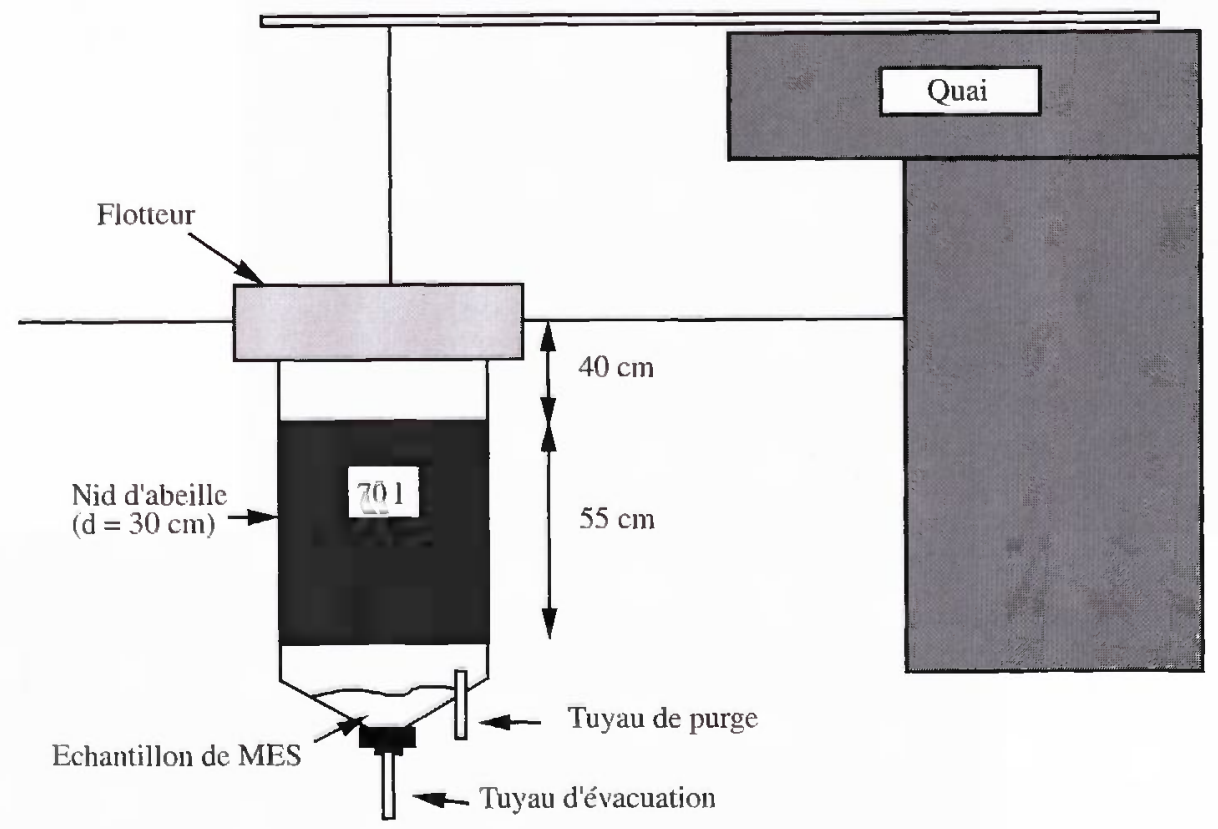

Fig. 2. - Schéma de principe d'une trappe à MES (exemple de la trappe du LABAM)

Fig. 2. - Scheme of a sediment trap (example : trap used by LABAM) 
nid d'abeilles; le diamètre de chaque structure hexagonale du nid est en général de l'ordre du centimètre. Au cours du prélèvement, la trappe est immergée et maintenue à une profondeur donnée par l'intermédiaire d'une bouée. A l'issue du prélèvement, la trappe est relevée verticalement; l'eau contenue à l'intérieur est évacuée par un système de purge et les MES décantées à la base de la trappe prélevées dans un petit volume d'eau. Le système de prélèvement, peu coûteux, présente l'avantage d'être autonome sur le long terme, les opérations de maintenance se réduisant à l'implantation du matériel et à son relevage en fin de période d'échantillonnage. La masse de matière recueillie, se présentant sous forme d'un concentrat utilisable pour l'ensemble des mesures classiquement souhaitées, peut cependant être faible, particulièrement si la vitesse du courant au point d'implantation est élevée. Les particules sont par ailleurs collectées selon leur propension à sédimenter. Aussi, il est probable que le système privilégie les particules les plus grossières, au détriment des particules fines ou des organismes vivants (phytoplancton); les spectres granulométrique et qualitatif des MES collectées seraient alors déplacés vers certaines fractions.

- Décanteur émergé (figure 3): l'eau de rivière est acheminée par l'intermédiaire d'une pompe péristaltique vers la base d'un décanteur composé de deux éléments placés en série : un décanteur lamellaire et un décanteur constitué d'une structure en nid d'abeilles. Le volume total des deux décanteurs réunis est de quelques dizaines de litres. Au cours du transfert

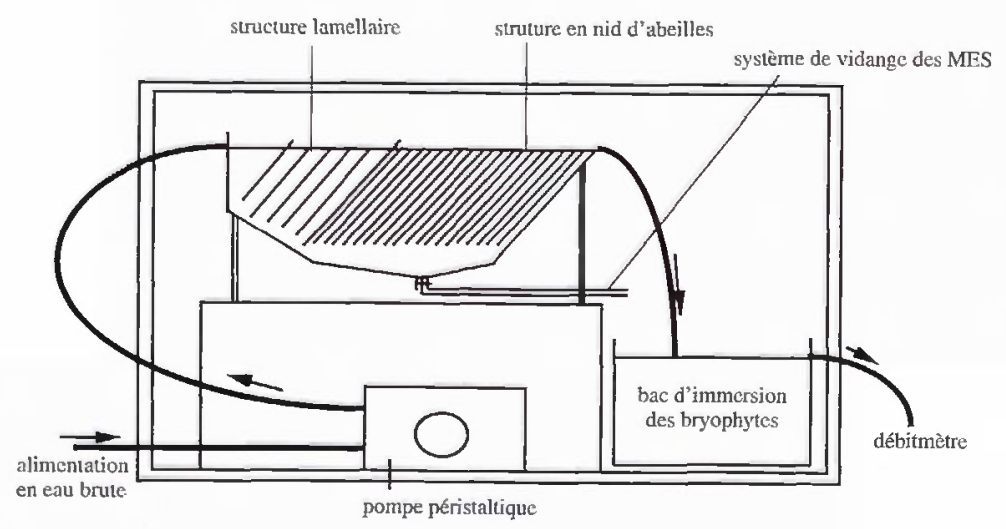

Fig. 3. - Schéma de principe d'un décanteur émergé (exemple du système EDF; le bac situé à l'aval a pour fonction l'implantation de bryophytes et est donc destiné à d'autres applications)

Fig. 3. - Scheme of an emerged decantor (example: EDF's system) 
de l'eau vers le sommet des décanteurs, les particules en suspension sédimentent sur les lames, puis sur le nid d'abeille. A l'issue du prélèvement, les MES concentrées peuvent être récupérées par un système de vidange situé à la base des décanteurs. Dans ce système, les MES sont collectées selon leur propension à sédimenter. Aussi, certains avantages et certaines limites précédemment évoqués pour les trappes immergées peuvent être repris ici : échantillonnage d'un concentrat de particules utilisable pour l'ensemble des mesures classiquement souhaitées, mais risque d'un déplacement des spectres granulométrique et qualitatif des MES collectées vers des fractions grossières. L'emploi d'un système émergé permet toutefois de s'affranchir des conditions hydrauliques du milieu et d'augmenter le temps de résidence de l'eau brute au sein du décanteur; la sédimentation peut donc être plus efficace que celle observée dans un décanteur immergé, et la masse d'échantillon récoltée est généralement nettement supérieure. Un temps de résidence trop long pourrait toutefois conduire à des échanges de métaux entre la phase dissoute et la phase particulaire au sein même du décanteur, conduisant à un enrichissement ou un appauvrissement métallique des MES finalement collectées. La maintenance de l'appareil est minime, et se réduit à quelques opérations en début et fin de période de prélèvement. Une source d'électricité constitue cependant une limite à l'utilisation généralisée de ce système.

D'autres techniques de prélèvement des MES peuvent être mises en œuvre: filtration tangentielle (Sansone, communication personnelle), filtration frontale immergée (Albrecht et al., 1995),... N'ayant pas été testées au cours des exercices d'intercomparaison présentés dans cette publication, leur principe ne sera pas détaillé.

\section{MATÉRIELS ET MÉTHODES}

\subsection{Description du site et pério- des de prélèvement}

Les prélèvements de MES ont été réalisés en rive droite de la Seine (à environ 3 mètres du bord), à une profondeur d'environ 0,5 mètre et à environ 900 mètres du point de rejet principal des eaux de refroidissement de la centrale de Nogent/Seine (point dénommé ci-après «Aval»). Au point de prélèvement, il y a théoriquement un bon mélange entre les eaux de la Seine et celles du rejet. Les eaux rejetées par la centrale sont généralement chargées en cuivre et zinc, dont une partie est sous forme particulaire (généralement, de 30 à $40 \%$ ); le point de prélèvement choisi constitue donc un site privilégié pour le suivi de ces polluants métalliques et l'inter- 
comparaison de plusieurs méthodes de prélèvement.

A titre complémentaire, plusieurs appareils (trappes, MIM) ont été également placés à lamont immédiat de la centrale, en rive droite de la Seine (point dénommé ci-après "Amont»). Les résultats obtenus en ce point fournissent des informations pour comparer les techniques de sédimentation-décantation dans le cas de teneurs en métaux proches des valeurs de référence.

Deux campagnes de prélèvement ont été réalisées au cours de deux périodes hydrologiques contrastées:

- 01-03 mars 1995: cette période correspond à une fin de crue, avec un fort débit de la Seine (262 à $285 \mathrm{~m}^{3} / \mathrm{s}$ ).

- 26-28 juin 1995 : cette période correspond à un début d'étiage, avec un débit moyen de la Seine (60 à $\left.62 \mathrm{~m}^{3} / \mathrm{s}\right)$.

\subsection{Appareils de prélèvement tes- tés}

Six appareils ont été utilisés lors des exercices d'intercomparaison: une centrifugeuse en continu; un système de prélèvement séquentiel d'eau, suivi d'une filtration frontale; un système de filtration sur cartouches; deux trappes immergées (trappe LABAM (Laboratoire de Bioélectrochimie et d'Analyse du Milieu) et trappe LGA (Laboratoire de Géologie Appliquée)); un décanteur émergé (dénommé MIM : «Module Intégrateur de Micropollution "). Les caractéristiques et les modes de fonctionnement de ces ap- pareils sont récapitulés dans le tableau 2. Au cours de ces deux campagnes, les divers appareils d'échantillonnage utilisés ont prélevés des MES de manière simultanée pendant 48 heures.

\subsection{Analyses physico-chimiques}

La concentration totale en $\mathrm{Cu}, \mathrm{Zn}$, $\mathrm{Fe}$ et $\mathrm{Mn}$ des divers échantillons est mesurée par Spectrométrie d'absorption atomique après minéralisation de ces derniers par attaque d'acides fluorhydrique et perchlorique concentrés. La spéciation des métaux particulaires est déterminée après des extractions sélectives selon la méthode de Tessier (Tessier et al., 1979]. Les protocoles relatifs à la mesure des métaux sont détaillés dans Taconnet, 1995.

La concentration en MES de l'eau de rivière est mesurée régulièrement après filtration sur un filtre prépesé de porosité $0,45 \mu \mathrm{m}$.

Les Matières Volatiles en Suspension (MVS) des échantillons prélevés par la centrifugeuse, les trappes ou le MIM sont déterminées sur des prises d'essai d'environ 1 gramme, chauffées à $550^{\circ} \mathrm{C}$ dans un four à moufle (Thermolyne, type 48000) pendant 5 heures. Les échantillons sont pesés avant et après calcination, après passage au dessicateur; la différence de masse permet de calculer leur teneur en MVS. Cette opération est réalisée en double pour tous les échantillons afin d'évaluer l'incertitude sur les résultats. Afin de déterminer 


\begin{tabular}{|c|c|c|c|c|c|c|c|c|}
\hline 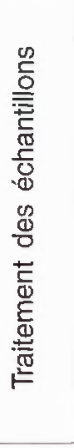 & 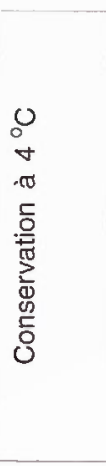 & & 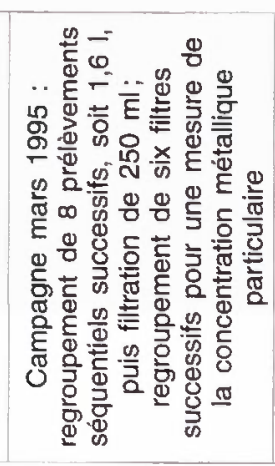 & 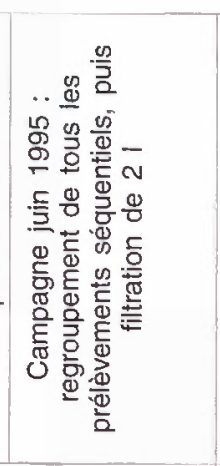 & 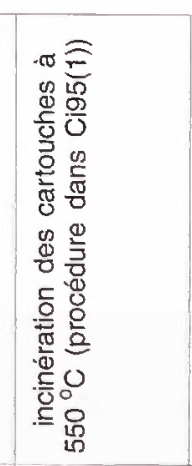 & 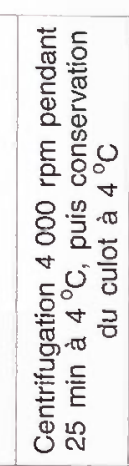 & 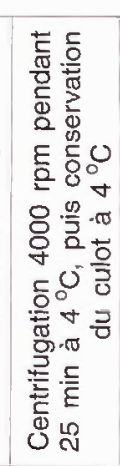 & 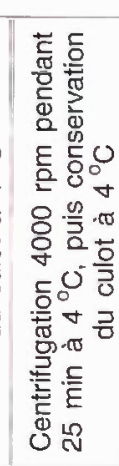 \\
\hline 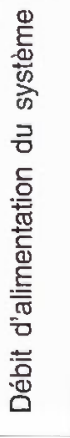 & 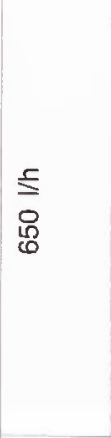 & 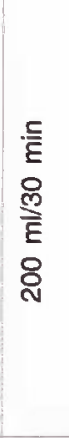 & & & 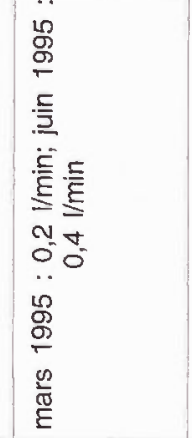 & & & $\frac{\underline{\underline{\underline{E}}}}{\underline{\underline{E}}}$ \\
\hline 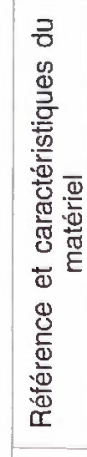 & 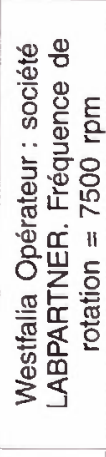 & 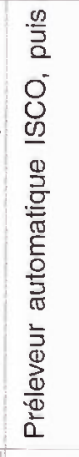 & 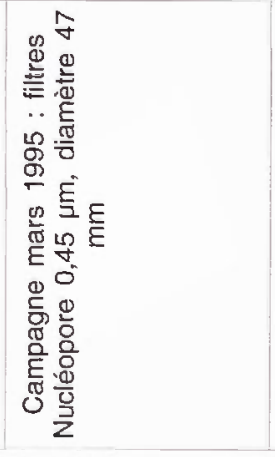 & 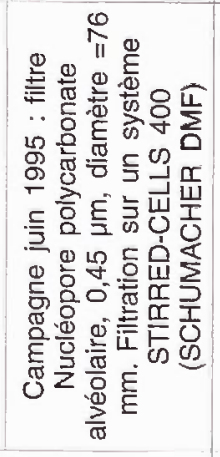 & 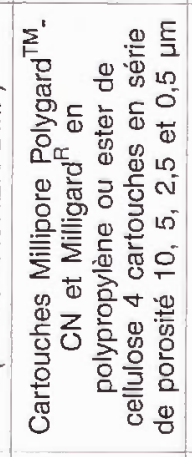 & 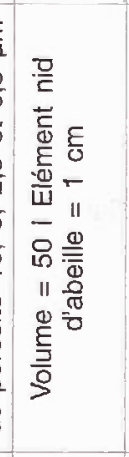 & 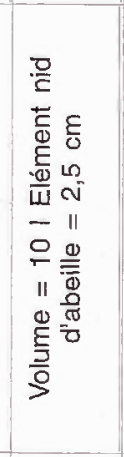 & 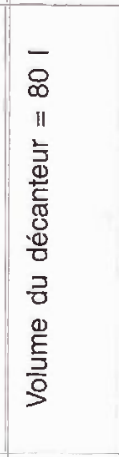 \\
\hline & 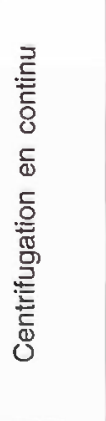 & 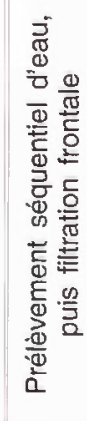 & & & 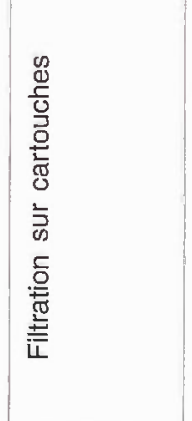 & 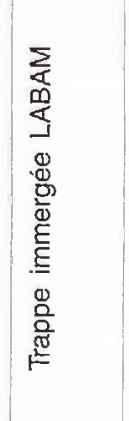 & 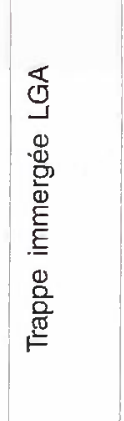 & 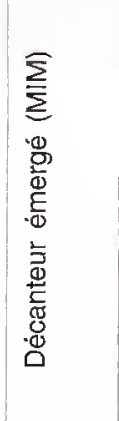 \\
\hline
\end{tabular}


les MVS de l'eau de rivière prélevée manuellement, les échantillons sont filtrés sur des filtres Whatman GFC prépesés en fibre de verre, de porosité 1,2 $\mu \mathrm{m}$. Ces derniers sont ensuite incinérés à $550^{\circ} \mathrm{C}$. La différence de masse des filtres avant et après calcination permet d'évaluer la teneur en MVS.

Le Carbone Organique Particulaire et le Carbone Inorganique Particulaire sont déterminés par un analyseur Beckman (Université de Bordeaux).

La chlorophylle a est dosée par spectrométrie d'absorption moléculaire, selon la norme AFNOR T90-117

Le spectre granulométrique des échantillons recueillis par la centrifugeuse, les trappes et le MIM est mesuré sur un granulomètre de type Coulter Counter LS 130 à diffraction laser, pouvant opérer de 0,1 à 900 $\mu \mathrm{m}$ et étalonné avec des micro sphères calibrées en latex. Les échantillons sont préalablement soumis à 90 secondes d'ultrasons, ayant pour effet de désagréger les particules et d'assurer une répétabilité satisfaisante des mesures.

\section{RÉSULTATS ET DISCUSSION}

\subsection{Conditions physico-chimiques du milieu et masses d'échan- tillons récoltées}

Les caractéristiques physico-chimiques au cours des deux campagnes d'intercomparaison sont synthétisées dans le tableau 3 . On notera que les caractéristiques physico-chimiques de l'écoulement sont restées assez stables au cours des suivis, avec des concentrations en MES totales représentatives d'un état moyen de la Seine.

La masse respective de MES recueillie au cours des 48 heures de suivi par les différentes techniques de prélèvement est fortement variable (de 0,6 à $620 \mathrm{~g} \mathrm{sec}$ en mars 1996) (tableau 4). II est important de noter que la quantité de matière récoltée par les trappes pour une même période d'intégration est fortement dépendante des conditions hydrauliques de l'écoulement. Ainsi, en juin 1995, les trappes ont recueilli une quantité de MES suffisante pour une large gamme d'analyses (de 25 à $50 \mathrm{~g}$ sec); au contraire, les masses prélevées en mars 1995, dans des conditions de débit plus élevé, sont beaucoup plus faibles (de 0,6 à $5 \mathrm{~g}$ sec). Ce point peut constituer une limite à l'utilisation des trappes pour le suivi des flux polluants en rivière, en particulier en période de crue. Le MIM, traitant une quantité d'eau déterminée indépendamment des conditions hydrauliques du cours d'eau, fournit des masses suffisantes pour une large gamme d'analyses (environ 250 grammes en 48 heures).

\subsection{Concentrations totales en mé- taux particulaires (figures 4 à 8 )}

Les “traceurs" permettant de comparer les techniques de prélèvement précédemment énumérées sont essentiellement constitués par les 
Tableau 3. - Caractéristiques physico-chimiques de la Seine au cours des deux campagnes

\begin{tabular}{|c|c|c|c|c|}
\hline Paramètres & \multicolumn{2}{|c|}{ Campagne 01-03 mars 1995 } & \multicolumn{2}{c|}{ Campagne 26-28 juin 1995 } \\
\hline & Point “Amont » & Point " Aval » & Point « Amont » & Point « Aval » \\
\hline Débit (en $\mathrm{m}^{3} . \mathrm{s}^{-1}$ ) & \multicolumn{2}{|c|}{$262-285$} & \multicolumn{2}{|c|}{$60-62$} \\
\hline MES (en mg. $\mathrm{l}^{-1}$ ) & $12,0 \pm 0,8$ & $12,2 \pm 1,1$ & $19,4 \pm 1,6$ & $17,1 \pm 1,4$ \\
\hline
\end{tabular}

Tableau 4. - Masses de MES recueillies au cours des deux campagnes

\begin{tabular}{|c|c|c|c|}
\hline Point de prélèvement & $\begin{array}{c}\text { Technique de } \\
\text { prélèvement }\end{array}$ & $\begin{array}{c}\text { Campagne de mars } \\
\text { 1995 - Masse de } \\
\text { MES recueillie } \\
\text { (en g sec) }\end{array}$ & $\begin{array}{c}\text { Campagne de juin } \\
\text { 1995 - Masse de } \\
\text { MES recueillie } \\
\text { (en g sec) }\end{array}$ \\
\hline \multirow{2}{*}{ Amont } & Trappe LABAM & $\approx 5$ & $\approx 50$ \\
\cline { 2 - 4 } & Trappe LGA & $\approx 0,8$ & $\approx 25$ \\
\hline \multirow{2}{*}{ Aval } & MIM & & $\approx 250$ \\
\hline & Trappe LABAM & $\approx 5$ & $\approx 50$ \\
\cline { 2 - 4 } & Trappe LGA & $\approx 0,6$ & $\approx 25$ \\
\cline { 2 - 4 } & MIM & $\approx 250$ & $\approx 250$ \\
\cline { 2 - 4 } & Centrifugeuse & $\approx 620$ & $\approx 480$ \\
\cline { 2 - 4 } & Cartouches & $\approx 10$ & $\approx 0,04$ \\
\hline
\end{tabular}

métaux rejetés par la centrale de Nogent/Seine, en l'occurrence le cuivre et le zinc; en plus de ces deux métaux traces, le fer et le manganèse ont également été analysés. Les comparaisons s'établissent sur les concentrations en métaux totaux mesurées dans les divers échantillons récoltés.

- ISCO et filtration : Lors de la première campagne d'intercomparaison, les concentrations en $\mathrm{Cu}$ et $\mathrm{Zn}$ mesurées sur les filtres après échantillonnage par ISCO apparaissent beaucoup plus élevées que celles ob- tenues à partir des autres méthodes de prélèvement. Une contamination de l'échantillon par ces deux éléments, imputable au matériel de I'ISCO ou au filtre lui-même, pourrait être à l'origine de ces surestimations. Les très fortes valeurs des concentrations en zinc, et surtout en cuivre, mesurées au point “Amont" apparaissent aberrantes et viennent donc appuyer l'hypothèse d'une contamination de l'échantillon par le matériel utilisé. En outre, la procédure expérimentale suivie lors de la $1^{\text {ère }}$ campa- 

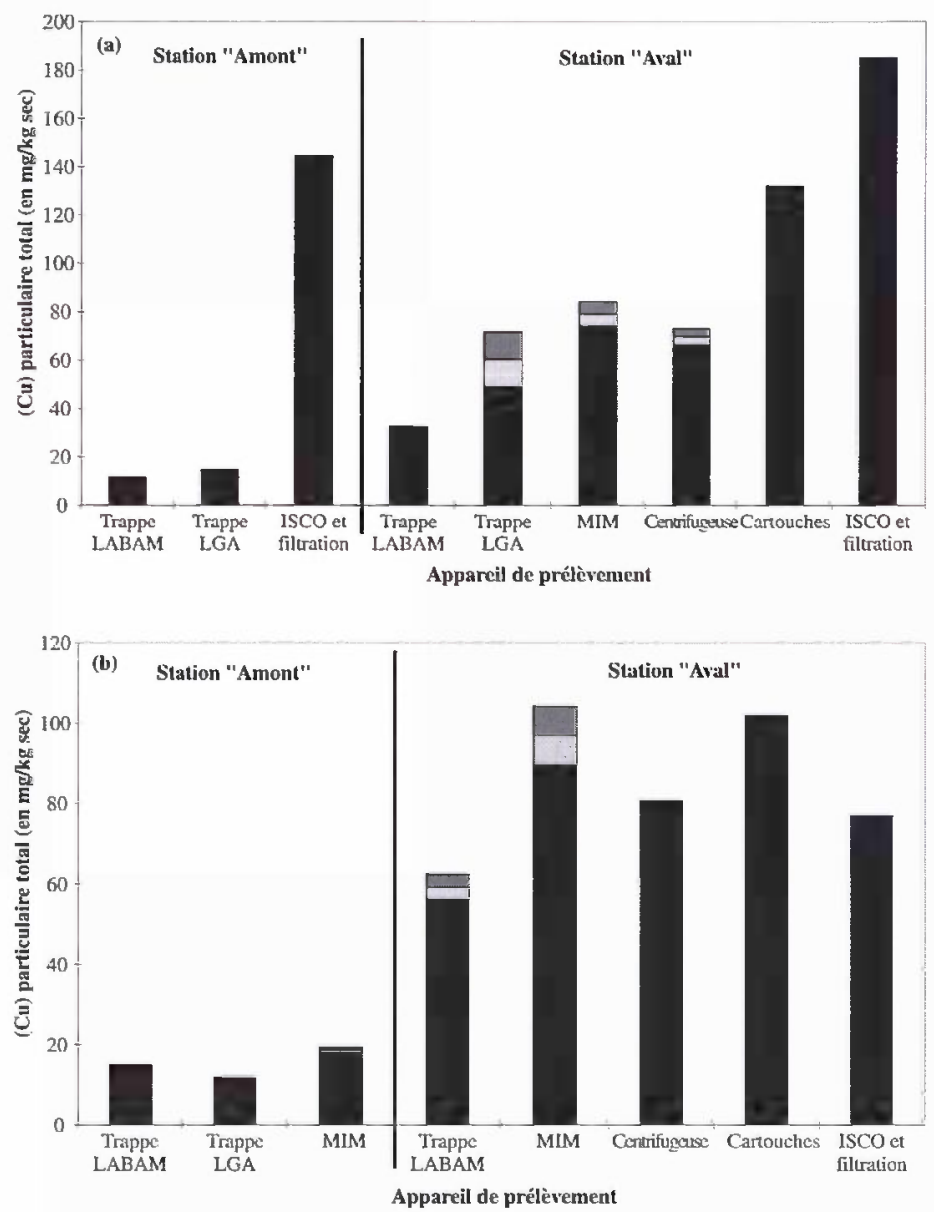

Fig. 4. - Mesures de la concentration totale en cuivre - (a) : campagne de mars 1995 - (b): campagne de juin 1995 (lorsque des duplicats de mesures ont été réalisés, sont représentées la valeur moyenne diminuée et augmentée de l'écart type)

Fig. 4. - Measurements of copper total concentration - (a) : March 1995 - (b) : June 1995

gne peut conduire à de fortes incertitudes: les masses de MES recueillies sur les filtres sont très faibles (3 mg par filtre); on aboutit alors à une forte dispersion des concentrations mesurées sur ces filtres, l'écart entre échantillons journaliers successifs étant supérieur à $80 \%$. Compte tenu des enseignements tirés de la $1^{\text {ère }}$ campagne, la procédure expérimentale mise en œuvre pour le système "ISCO+filtration» a été modifié lors de la $2^{\text {nde }}$ campagne (changement du type de filtre et du système de filtration; filtration d'un volume plus important); la nouvelle procédure 

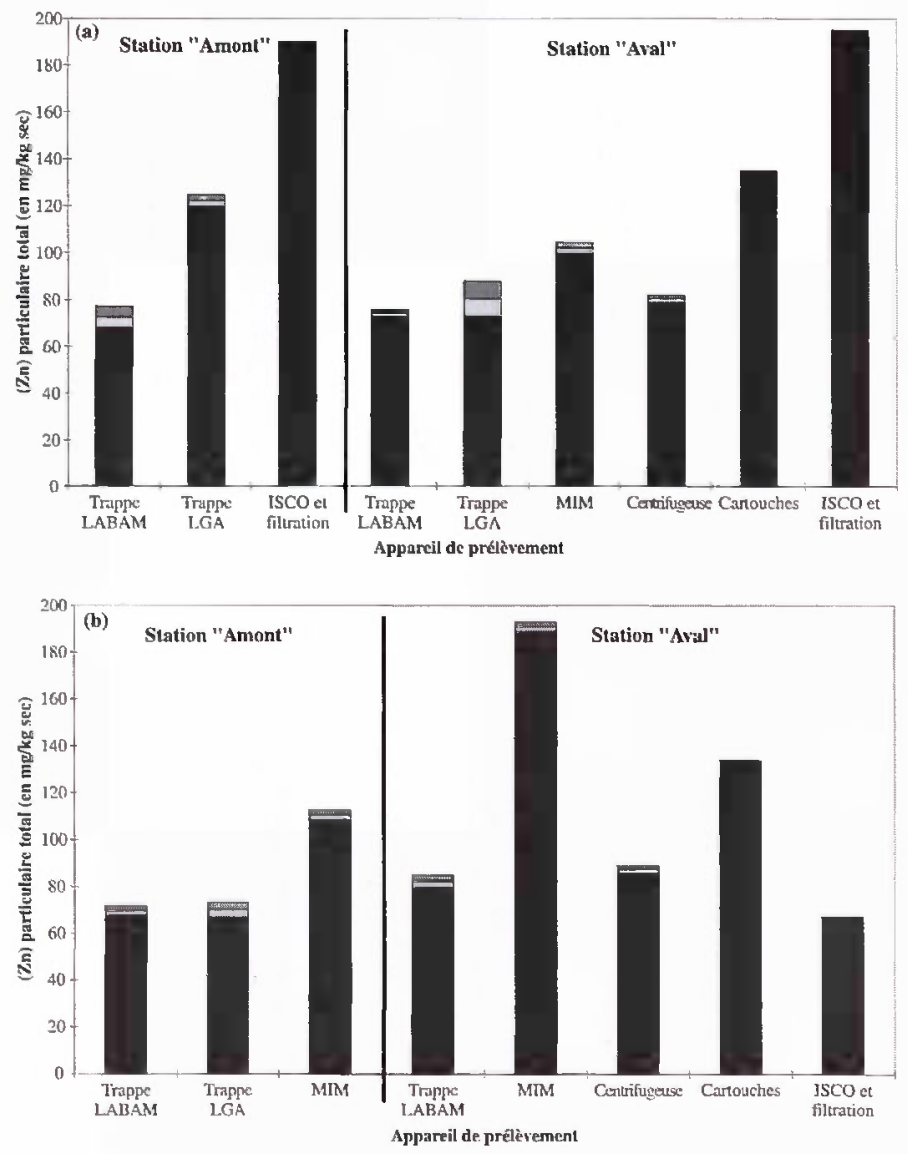

Fig. 5. - Mesures de la concentration totale en zinc - (a) : campagne de mars 1995 - (b): campagne de juin 1995 (lorsque des duplicats de mesures ont été réalisés, sont représentées la valeur moyenne diminuée et augmentée de l'écart type)

Fig. 5. - Measurements of zinc total concentration - (a) : March 1995 - (b) : June 1995

conduit à des écarts et des incertitudes moindres.

- Cartouches filtrantes: L'utilisation des cartouches filtrantes aboutit généralement à des concentrations métalliques supérieures à la médiane, calculée en tenant compte des mesures réalisées sur tous les échan- tillons collectés. Ces surestimations pourraient être dues au piégeage par le filtre lui-même des métaux qui le traverse sous forme colloïdale ou dissoute. II convient toutefois de signaler que de fortes incertitudes pèsent sur les résultats de la $1^{\text {ère }}$ campagne : en raison de problèmes intervenus lors 

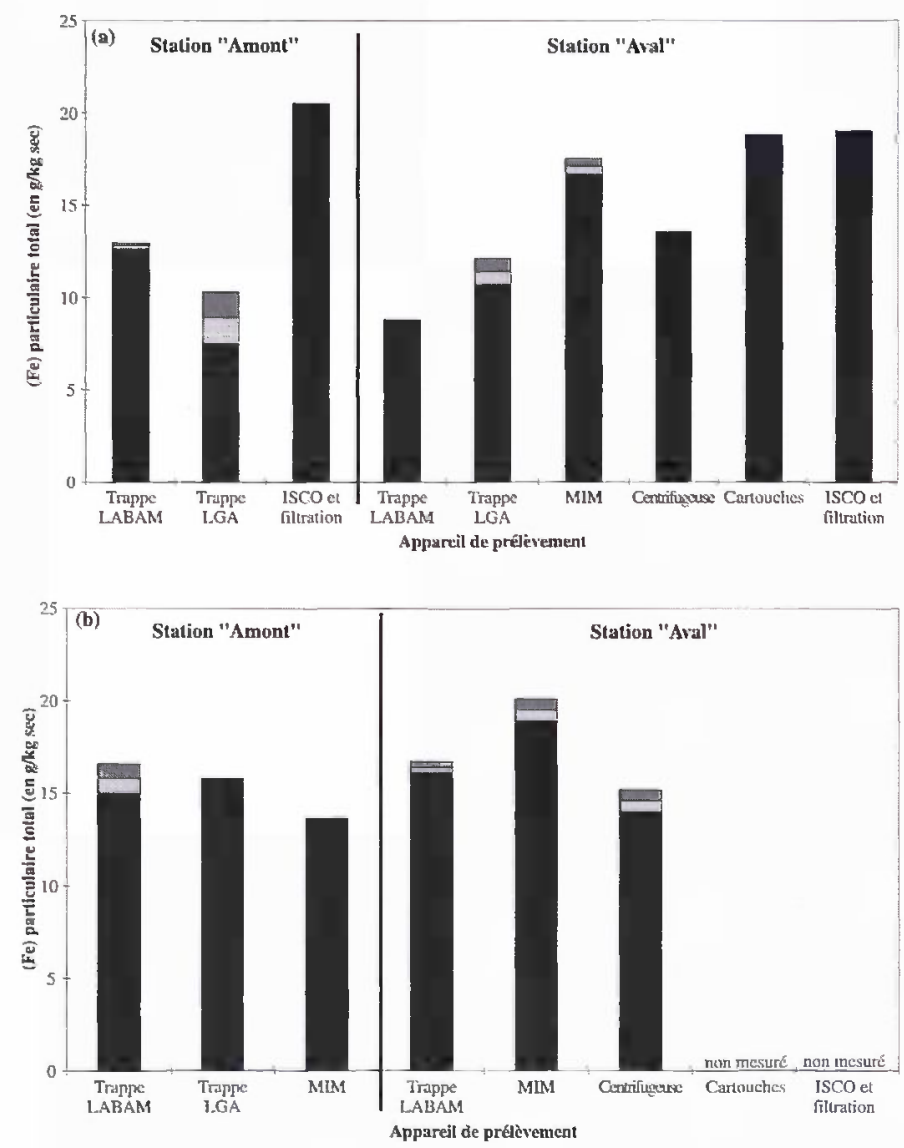

Fig. 6. - Mesures de la concentration totale en fer - (a) : campagne de mars 1995 - (b) : campagne de juin 1995 (lorsque des duplicats de mesures ont été réalisés, sont représentées la valeur moyenne diminuée et augmentée de l'écart type)

Fig. 6. - Measurements of iron total concentration - (a) : March 1995 - (b) : June 1995

de la filtration, les masses sèches de MES recueillies n'ont pu être estimées qu'avec une «précision» de $40 \%$.

- Trappes immergées: L'utilisation de deux trappes différentes au cours de deux périodes hydrologiques contrastées permet d'obtenir des infor- mations sur les conditions d'utilisation de cette méthode de prélèvement:

$\rightarrow$ la reproductibilité de la méthode peut, sous certaines conditions, être médiocre. Ainsi, deux trappes de géométries différentes, placées à quelques mètres de distance, et utilisées au cours d'une même période, 

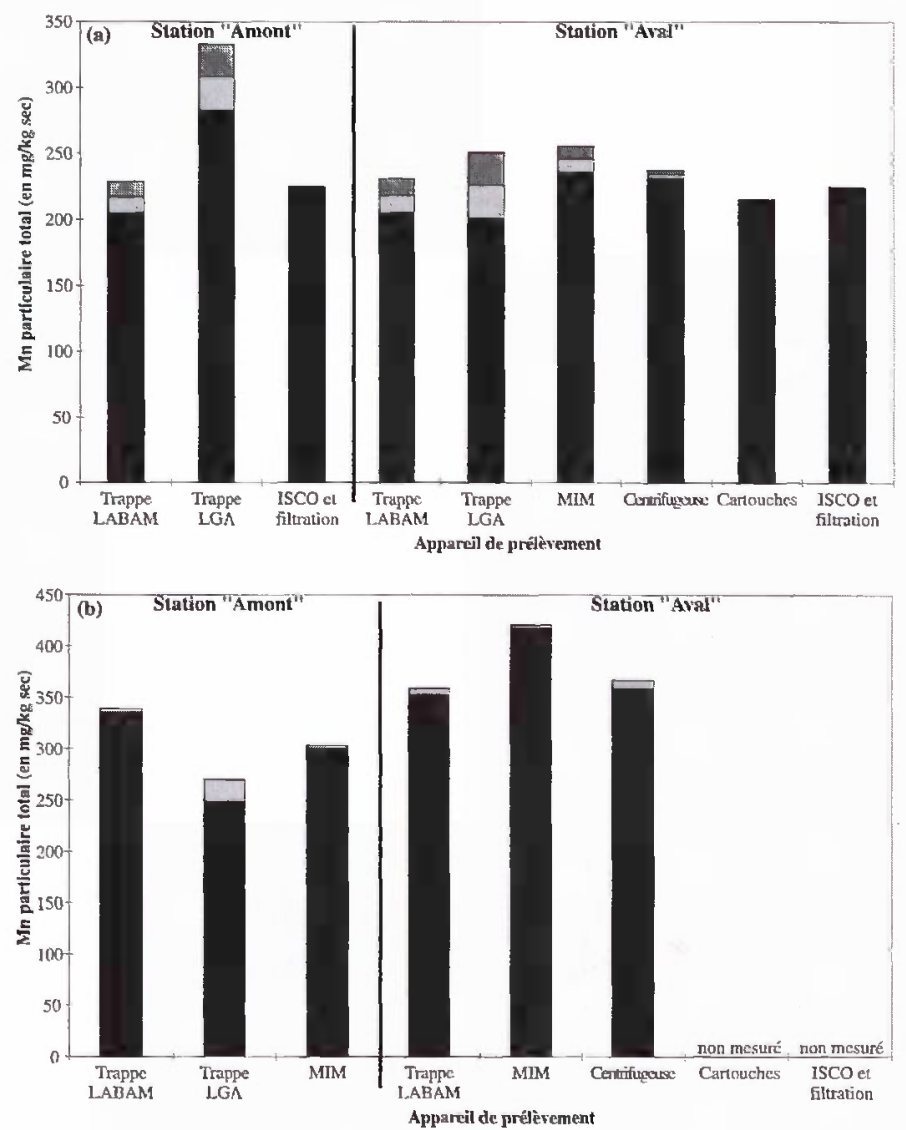

Fig. 7. - Mesures de la concentration totale en manganèse - (a) : campagne de mars 1995 (b) : campagne de juin 1995 (Iorsque des duplicats de mesures ont été réalisés, sont représentées la valeur moyenne diminuée et augmentée de l'écart type)

Fig. 7. - Measurements of manganese total concentration - (a) : March 1995 - (b) : June 1995

aboutissent à des résultats qui peuvent être significativement différents pour certains éléments. A titre d'exemple, un facteur 2 sépare les concentrations en cuivre mesurées à la station "Aval» sur les échantillons respectivement collectés par la trappe LGA et la trappe LABAM; les concen- trations en manganèse et zinc mesurées au point "Amont " au cours de la $1^{\text {ère }}$ campagne présentent des écarts de 35 à $50 \%$ entre les échantillons recueillis par les deux trappes.

$\rightarrow$ la reproductibilité du prélèvement et les écarts de concentrations par rapport aux autres techniques 

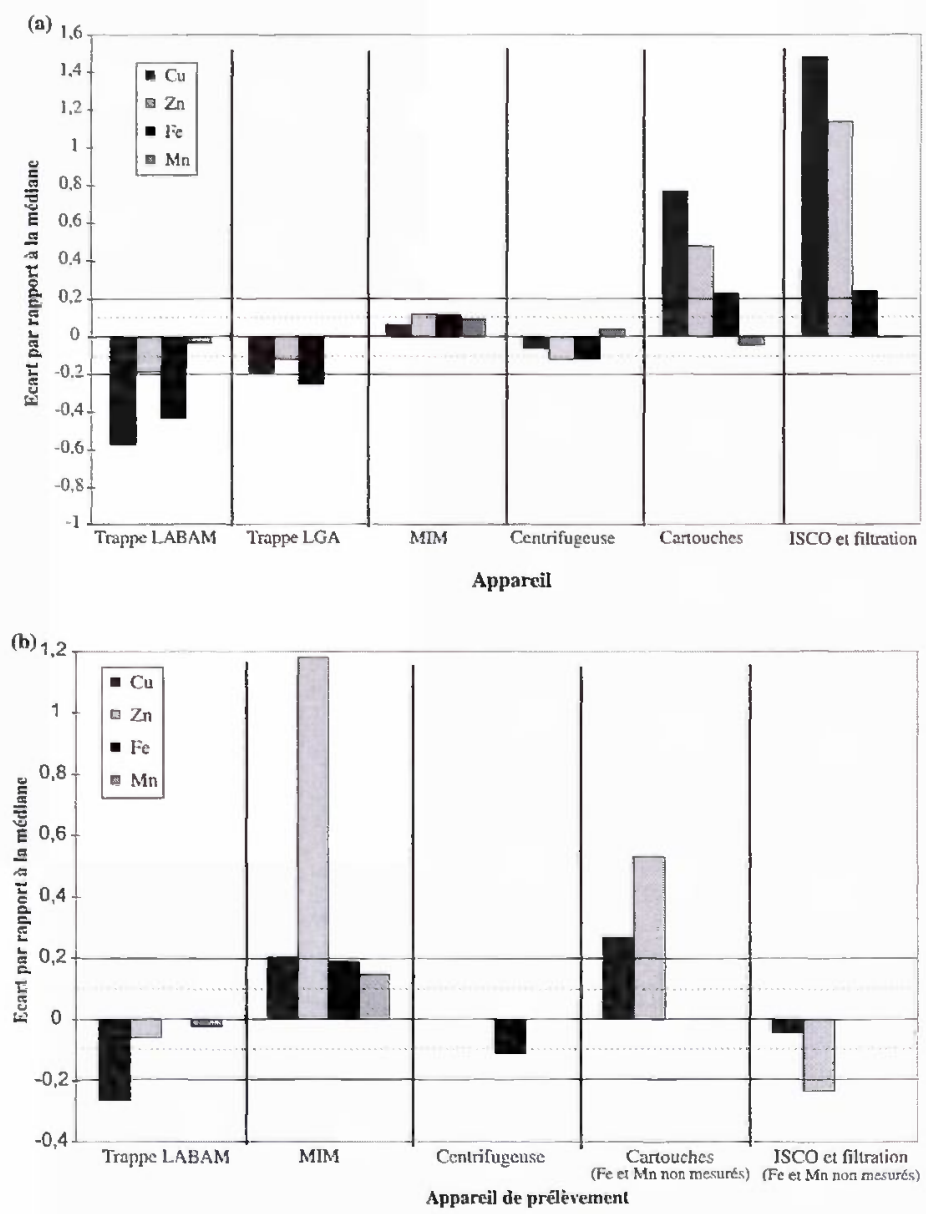

Fig. 8. - Ecarts par rapport à la médiane pour les différentes mesures réalisées au point «Aval » - (a) : campagne de mars 1995 - (b) : campagne de juin 1995

Fig. 8. - Differences between single measurements and the mediane - Station "Downstream (a): March 1995 - (b) : June 1995

semblent dépendre des conditions hydrologiques rencontrées au cours de la période d'échantillonnage. Ainsi, alors que des écarts significatifs avaient été observés entre les deux trappes lors de la $1^{\text {ère }}$ campagne d'intercomparaison, réalisée en période de crue, les mesures réalisées sur les échantillons collectés par les deux trappes au point «Amont» lors de la $2^{\text {nde }}$ campagne apparaissent similaires. Par ailleurs, les écarts de concentration par rapport à la médiane, déterminés en tenant compte de l'en- 
semble des mesures réalisées au point «Aval ", sont plus faibles en période d'étiage.

$\rightarrow$ la très faible masse de matériel particulaire recueillie au cours de certains prélèvements (cas de la trappe LGA au cours de la $1^{\text {ère }}$ campagne) provoque dans certains cas une forte dispersion entre duplicats (environ $20 \%$ ), probablement due à la difficulté d'obtenir un aliquot homogène.

- Décanteur émergé (MIM) : Bien qu'à l'instar des trappes immergées, le prélèvement des MES s'effectue selon la propension de ces dernières à sédimenter, les résultats obtenus apparaissent dans certains cas significativement différents. Ainsi, des écarts supérieurs à $30 \%$ sont régulièrement observés entre les concen- trations mesurées sur les échantillons collectés respectivement par les trappes et le MIM. Au contraire, les analyses issues du MIM apparaissent en général en accord avec celles issues de la centrifugeuse. Seule la mesure du zinc particulaire au cours de la $2^{\text {nde }}$ campagne $d^{\prime}$ intercomparaison s'écarte de la médiane. Par ailleurs, au cours de la $2^{\text {nde }}$ campagne, trois MIM ont été respectivement implantés au point "Amont", dans le rejet de la centrale et au point "Aval». Les mesures de métaux réalisées sur les échantillons collectés en ces 3 points permettent d'établir des bilans de flux métalliques particulaires et de vérifier ainsi la cohérence des prélèvements effectués en différents points par le MIM (figure 9). On note que le flux de cuivre déterminé à l'aval de la cen-

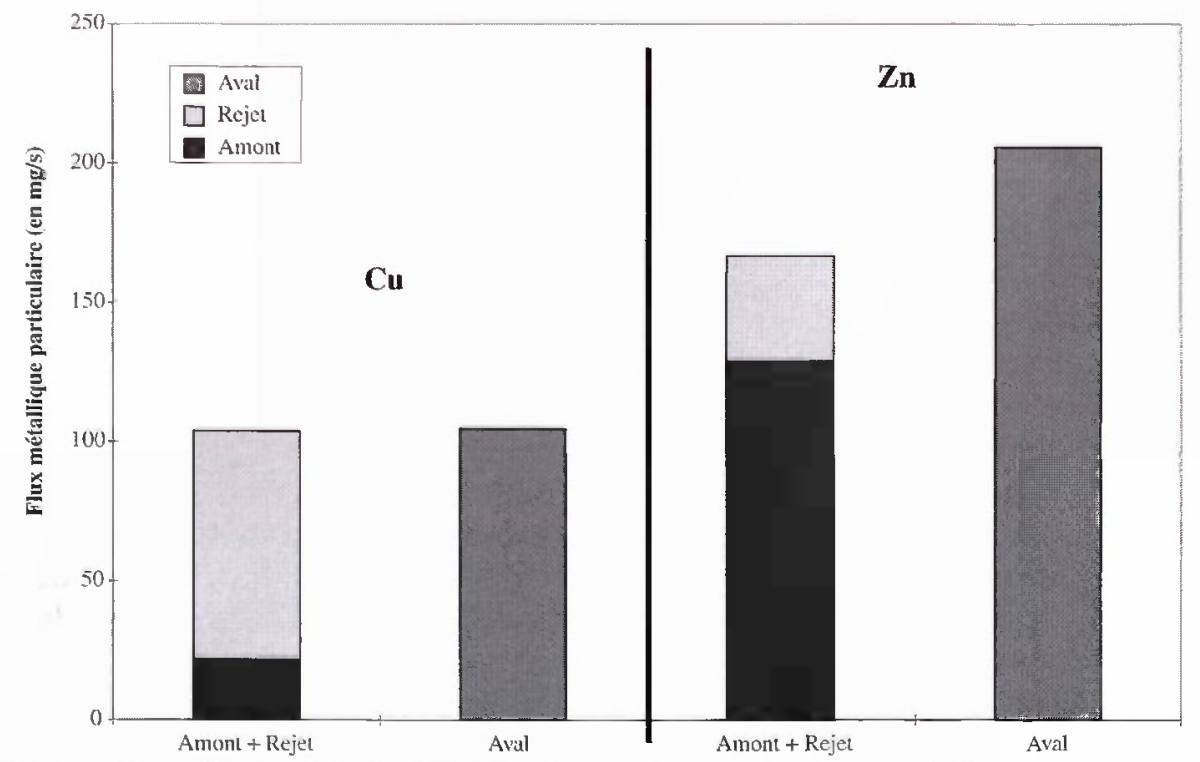

Fig. 9. - Flux métalliques particulaires établis à partir des mesures sur MIM

Fig. 9. - Flux of particulate metals determined by measurements on samples collected by the emerged decantor 
trale est égal au flux obtenu en additionnant les apports provenant de l'amont et du rejet; pour le zinc, les flux respectivement déterminé à l'aval et calculé en tenant compte des apports issus de l'amont et du rejet présentent un écart d'environ $20 \%$. Les bilans effectués avec les MIM implantés aux diverses stations de prélèvement apparaissent donc cohérents et la méthode utilisée pourrait être adaptée à l'évaluation sur le long terme des flux de cuivre particulaire issus de l'installation.

- Centrifugeuse: Les mesures réalisées sur les échantillons collectés par la centrifugeuse aboutissent généralement à des valeurs proches de la médiane de l'ensemble des analyses métalliques.

\subsection{Analyse granulométrique}

L'un des critères essentiels permettant de juger de la représentativité d'un échantillonnage de MES repose sur le respect du spectre granulométrique des particules présentes dans le milieu naturel. II importe donc de connaître la taille des MES préférentiellement récoltées par les diverses techniques de prélèvement et la répartition des métaux par classes granulométriques.

- Granulométrie des échantillons: L'analyse des déciles, des quartiles et de la médiane des différents spectres granulométriques fait apparaître de fortes différences entre les divers échantillons récoltés lors de la $1^{\text {ère }}$ campagne d'intercomparaison, réalisée en période de crue (tableau 5).

Tableau 5. - Caractéristiques des spectres granulométriques: $1^{\text {ère }}$ campagne de mesures

\begin{tabular}{|c|c|c|c|c|c|c|c|c|}
\hline \multirow[t]{2}{*}{ Point } & \multirow[t]{2}{*}{ Technique } & \multirow[t]{2}{*}{$\begin{array}{l}\mathrm{Nb} \text { de } \\
\text { mesures }\end{array}$} & \multirow[t]{2}{*}{$\begin{array}{c}\text { Reproducti- } \\
\text { bilité }\end{array}$} & \multicolumn{5}{|c|}{ Diamètres correspondant aux déciles (en $\mu \mathrm{m}$ ) } \\
\hline & & & & $10 \%$ & $25 \%$ & $50 \%$ & $75 \%$ & $90 \%$ \\
\hline \multirow[t]{2}{*}{ "Amont $»$} & $\begin{array}{c}\text { Trappe } \\
\text { LABAM }^{*}\end{array}$ & 2 & $\begin{array}{c}\text { Très } \\
\text { mauvaise }\end{array}$ & 1,3 & 4,7 & 12,3 & 45 & 112 \\
\hline & $\begin{array}{c}\text { Trappe } \\
\text { LGA }\end{array}$ & 1 & $\begin{array}{c}\text { non } \\
\text { déterminée }\end{array}$ & 4,4 & 13 & 25,9 & 53 & 146 \\
\hline \multirow[t]{4}{*}{ "Aval » } & $\begin{array}{c}\text { Trappe } \\
\text { LABAM }^{*}\end{array}$ & 3 & Bonne & 2,8 & 10,5 & 52 & 230 & 379 \\
\hline & $\begin{array}{c}\text { Trappe } \\
\text { LGA }\end{array}$ & 2 & Bonne & 3,8 & 11 & 22,8 & 49,4 & 134,9 \\
\hline & MIM & 4 & $\begin{array}{c}\text { Très } \\
\text { bonne }\end{array}$ & 1,6 & 5,5 & 12,3 & 27,4 & 53,6 \\
\hline & $\begin{array}{c}\text { Centrifugeu } \\
\text { se }\end{array}$ & 3 & $\begin{array}{l}\text { Assez } \\
\text { bonne }\end{array}$ & 0,6 & 1,6 & 4,8 & 15,7 & 38,9 \\
\hline
\end{tabular}

* échantillons préalablement centrifugés au laboratoire. 
Tableau 6. - Caractéristiques des spectres granulométriques : $2^{\text {nde }}$ campagne de mesures

\begin{tabular}{|c|c|c|c|c|c|c|c|c|}
\hline Point & Technique & $\begin{array}{c}\text { Nb de } \\
\text { mesures }\end{array}$ & $\begin{array}{c}\text { Reproduc- } \\
\text { tibilité }\end{array}$ & \multicolumn{3}{|c|}{ Diamètres correspondant aux déciles (en $\mu \mathrm{m})$} \\
\hline " Amont " & $\begin{array}{c}\text { Trappe } \\
\text { LABAM* }\end{array}$ & 3 & Médiocre & 3,1 & 4,23 & 10,8 & 23,3 & 42,1 \\
\hline $\begin{array}{c}\text { Trappe } \\
\text { LGA }\end{array}$ & 3 & $\begin{array}{c}\text { Assez } \\
\text { bonne }\end{array}$ & 5,1 & 9,4 & 19,6 & 31,4 & 41,8 \\
\hline "Aval » & $\begin{array}{c}\text { MIM } \\
\begin{array}{c}\text { Trappe } \\
\text { LABAM* }\end{array}\end{array}$ & 3 & Très bonne & 4,7 & 8,1 & 15,3 & 28,4 & 40,3 \\
\hline $\begin{array}{c}\text { Trappe } \\
\text { LGA }\end{array}$ & 3 & Acceptable & 3 & 4,8 & 9,6 & 18,5 & 31,3 \\
\hline $\begin{array}{c}\text { MIM } \\
\text { Centrifu- } \\
\text { geuse }\end{array}$ & 3 & Bonne & 5,4 & 9,3 & 17,3 & 31,2 & 47,6 \\
\hline
\end{tabular}

* échantillons préalalement centrifugés au laboratoire

Les points suivant ont ainsi été mis en évidence :

$\rightarrow$ La reproductibilité de l'échantillonnage par trappe apparaît médiocre. Ainsi, les MES recueillies par la trappe LABAM respectivement aux points "Amont» et "Aval» sont de granulométries fortement différentes: la contribution des sables grossiers supérieurs est beaucoup plus importante au point «Aval» (25\% des particules sont de taille supérieure à $230 \mu \mathrm{m})$. Les deux points de prélèvement étant distants de quelques centaines de mètres seulement, il est improbable qu'un changement de la granulométrie des particules entre les deux stations soit responsable de telles différences. De même, les particules respectivement recueillies par les trappes LABAM et LGA, placées à quelques mètres de distance l'une de l'autre, présentent des spectres granulométriques fortement différents: le diamètre médian des MES collectées par la trappe LABAM est environ 2 fois supérieur à celui des MES échantillonnées par la trappe $\mathrm{LGA}^{(1)}$. II apparaît donc qu'en période de crue, la géométrie de la trappe et/ou les conditions locales d'écoule. ment influencent fortement l'échantillonnage par trappe.

(1) II convient toutefois de signaler que les échantillons récoltés par la trappe LABAM ont été préalablement centrifugé au laboratoire et que cette préparation a pu provoquer une modification de la granulométrie. 
$\rightarrow$ Bien que conçus selon le même principe de prélèvement, en l'occurrence la sédimentation-décantation des particules, les trappes et le MIM collectent des MES présentant des granulométries fortement différentes. Ainsi, par exemple, d'après les valeurs des déciles, des quartiles et de la médiane, on peut estimer que la trappe LGA récolte des particules environ deux fois plus grosses que le MIM.

$\rightarrow$ Le spectre granulométrique de l'échantillon recueilli par la centrifugeuse montre une forte contribution des particules les plus fines (la moitié des particules ont un diamètre inférieur à $5 \mu \mathrm{m}$ ). II est toutefois impossible d'évaluer les modifications apportées à la granulométrie des MES lors du prélèvement, à la suite du stress mécanique induit par la centrifugation (éclatement des particules, agrégation de colloïdes,...).

Les résultats acquis lors de la $2^{\text {ème }}$ campagne d'intercomparaison, réalisée en période d'étiage, permettent d'observer les points suivants:

$\rightarrow$ Les particules recueillies par les trappes sont beaucoup moins grossières qu'en période de crue. La reproductibilité de l'échantillonnage apparaît par ailleurs de meilleure qualité. $\rightarrow$ les spectres obtenus sur les échantillons récoltés par les trappes et le MIM, tant au point «Amont» qu'au point «Aval», apparaissent proches.

- Répartition des métaux par classes granulométriques: La répartition des métaux entre différentes classes granulométriques peut être approchée par l'utilisation de cartouches filtrantes de différentes porosités (cf Techniques de prélèvement). Ainsi, au cours des deux expériences d'intercomparaison, l'emploi de 4 cartouches placées en série a permis d'extraire sélectivement les particules appartenant à 4 classes granulométriques distinctes : > $10 \mu \mathrm{m}, 5-10 \mu \mathrm{m}$, 2,5-5 $\mu \mathrm{m}$ et $0,5-2,5 \mu \mathrm{m}$. La procédure expérimentale suivie permet ainsi d'aboutir à des concentrations métalliques spécifiques aux classes granulométriques extraites $^{(2)}$. Les résultats obtenus (figure 10) montrent que le cuivre présente une affinité différente vis à vis des particules selon la taille de ces dernières. Ainsi, les particules supérieures à $10 \mu \mathrm{m}$ fixent de 3 à 5 fois moins de cuivre que les particules dont la taille est comprise entre 2,5 et $10 \mu \mathrm{m}$. II semble par ailleurs que la capacité de fixation du cuivre décroisse pour les particules les plus fines. Les effets granulométriques

(2) Les concentrations sont exprimées en mg. $\mathrm{kg}^{-1}$ cendres, et ne peuvent être converties en mg. $\mathrm{kg}^{-1} \mathrm{sec}$, unitè plus classiquement utilisée. Toutefois, la teneur en matière organique étant relativement faible au cours des deux campagnes (entre 10 et $20 \%$ des MES totales), on peut estimer que les résultats obtenus fournissent une approximation correcte de la répartition des métaux entre les différentes classes granulométriques, même s'ils sont exprimés dans une unité peu classique. 

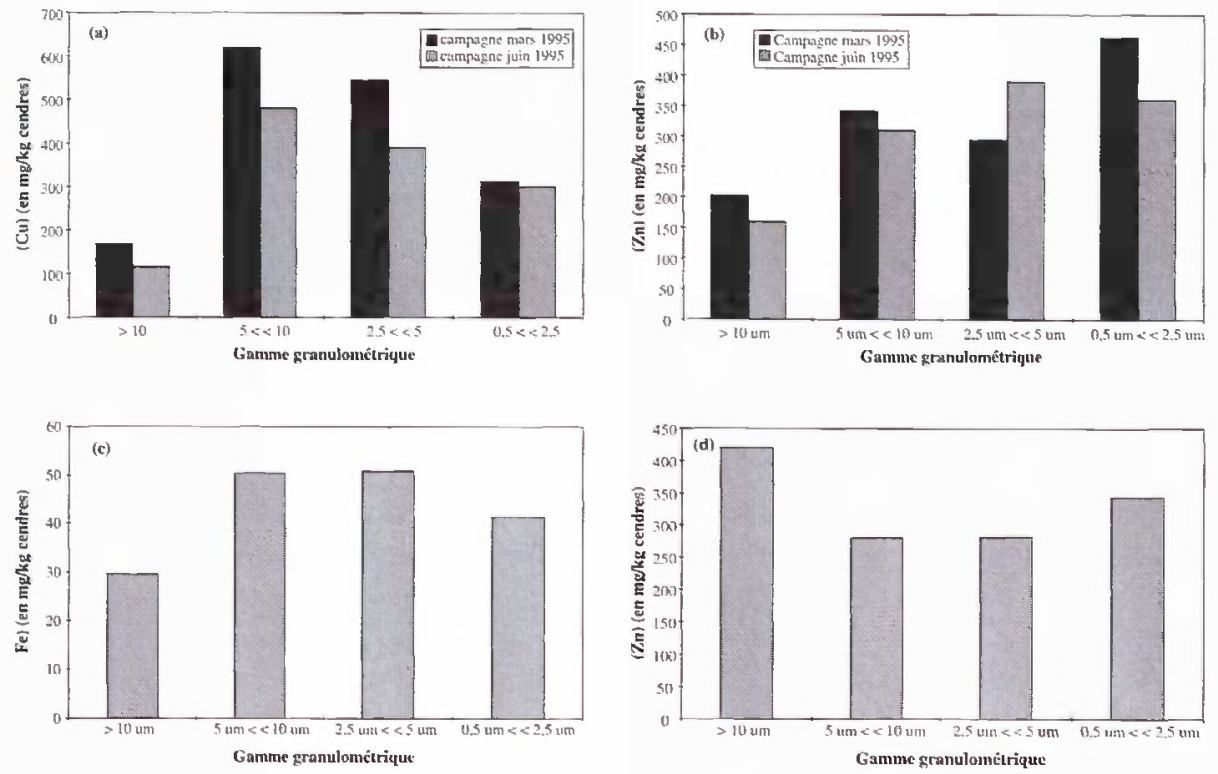

Fig. 10. - Répartition des métaux par classes granulométriques - (a) : cuivre ; (b) : zinc; (c) : fer; (d) : manganèse

Fig. 10. - Metals distribution among different granulometric classes - (a) : copper; (b) : zinc; (c) : iron; (d): manganese

apparaissent moins marqués pour le zinc. De fait, le rapport entre les concentrations mesurées sur les cartouches de porosité supérieure ou inférieure à $10 \mu \mathrm{m}$ est de l'ordre de 2 à 3 . Les MES qui présentent la plus forte affinité pour le fer sont comprises entre 2,5 et $10 \mu \mathrm{m}$. Le manganèse présente quant à lui un comportement particulier, les particules les plus grosses ayant l'affinité la plus élevée pour cet élément.

- Normalisation granulométrique: La combinaison des résultats établissant le spectre granulométrique des divers échantillons et la répartition des métaux par classes granulométriques permet d'avancer des hypo- thèses cohérentes expliquant les différences observées sur les mesures de métaux totaux. Ainsi, lors de la $1^{\text {ère }}$ campagne d'intercomparaison, il est apparu que le cuivre, et à un degré moindre le fer, étaient préférentiellement associés aux particules comprises entre 2,5 et $10 \mu \mathrm{m}$. Or, la proportion de particules appartenant à cette classe dépend fortement de l'échantillon considéré; on peut ainsi établir l'ordre croissant suivant: Trappe LABAM < Trappe LGA < Centrifugeuse < MIM. Les concentrations particulaires totales en cuivre et fer suivent le même ordre: $(\mathrm{Cu})_{\text {trappe LABAM }}<$ $(\mathrm{Cu})_{\text {trappe }}$ LGA $<(\mathrm{Cu})_{\text {centrifugeuse }}<$ (Cu) Mim. Pour le cuivre et le fer, les 
mesures de métal particulaire total doivent donc être corrigées afin de tenir compte de la variabilité du spectre granulométrique entre échantillons: les mesures brutes peuvent être normalisées en considérant la proportion de particules dans les classes 0,5-2,5 $\mu \mathrm{m}, 2,5-5 \mu \mathrm{m}, 5-10 \mu \mathrm{m}$ et 10-60 $\mu^{(3)}$, et les concentrations métalliques estimées pour chacune de ces classes (cf figure 10). Cette méthode permet par exemple de corriger les mesures issues de l'échantillon collecté par la trappe LABAM, qui présentait une proportion en particules grossières élevée : alors que les mesures brutes en cuivre et fer issues de la trappe LABAM étaient fortement inférieures à celles obtenues par les autres techniques, les concentrations corrigées sont du même ordre de grandeur (figure 11). Cet exemple montre que la normalisation granulométrique permet de réduire les écarts de mesures entre échantillons et que, par conséquent, le déplacement des spectres granulométriques est en grande partie responsable des différences inter-

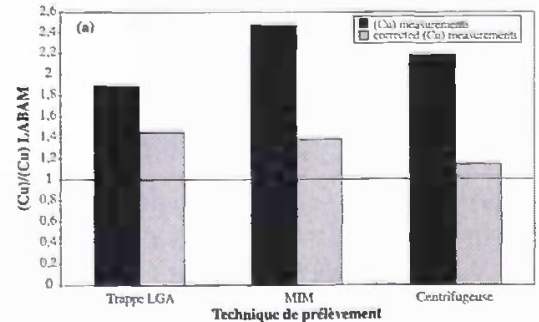

échantillons observées pour le cuivre et le fer. L'effet granulométrique est moins marqué lors de la $2^{\text {ème }}$ campagne d'intercomparaison, les trappes LABAM ou LGA ayant collecté un matériel plus fin.

\subsection{Matière organique et inorganique}

Les métaux présentent une affinité différente vis à vis de la matière particulaire selon la nature chimique de cette dernière. La matière organique peut en particulier jouer un rôle important pour certains éléments, tels que le cuivre. C'est la raison pour laquelle des mesures ont été entreprises afin d'évaluer la teneur en matière organique et inorganique des différents échantillons prélevés. La teneur en matière organique est estimée par 3 paramètres: les MVS, le COP et la chlorophylle a (cf Matériels et méthodes). Des mesures de carbone inorganique particulaire (CIP) ont également été réalisées. Les ré-

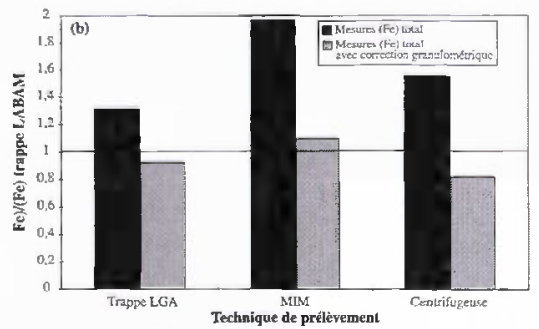

Fig. 11. - Comparaison des mesures issues de la trappe LABAM et des autres techniques avec ou sans correction granulométrique; (a) : cuivre, mars 1995 ; (b) : fer, mars 1995.

Fig. 11. - Comparison between measurements on samples collected by the LABAM's trap and the other methods respectively, with or without granulometric correction; (a) : copper, March 1995 ; (b) : iron, March 1995

(3) Les particules supérieures à $60 \mu \mathrm{m}$ sont supposées ne pas interagir avec les métaux. 
sultats des diverses analyses réalisées sont synthétisées dans les tableaux 7 et 8 .

Les mesures réalisées lors de la première campagne d'intercomparaison semblent indiquer des différences significatives dans les teneurs en matière organique des différents échantillons. Ainsi, l'échantillon recueilli par le MIM présente une teneur en MVS 2 fois plus élevée que celui récolté par la trappe LABAM. Ces observations peuvent être reliées aux analyses granulométriques: les grosses particules collectées par la trappe sont probablement de nature minérale et "diluent " la teneur en matière organique de l'échantillon.

Tableau 7. - Mesures de matière organique particulaire réalisées lors de la campagne de mars 1995

\begin{tabular}{|c|c|c|c|}
\hline Point & $\begin{array}{c}\text { Technique de } \\
\text { prélèvement }\end{array}$ & MVS (\% MES totales) & COP $\left(\mathrm{mg}^{\mathrm{k}} \mathrm{kg}^{-1} \mathrm{sec}\right)$ \\
\hline "Amont " & Trappe LABAM & $6,2 \pm 0,1$ & non réalisé \\
\hline "Ava " & Centrifugeuse & $6,6 \pm 0,04$ & non réalisé \\
\hline & Trappe LABAM & $4,8 \pm 0,1$ & non réalisé \\
\hline & MIM & $10,9 \pm 0,06$ & 3,4 \\
\hline & $\begin{array}{c}\text { prélèvement manuel } \\
\text { et filtration }\end{array}$ & non réalisé & $5,6 \pm 0,6$ \\
\hline
\end{tabular}

Tableau 8. - Mesures matière organique particulaire réalisées lors de la campagne 26-28 juin 1995

\begin{tabular}{|c|c|c|c|c|c|}
\hline Point & $\begin{array}{l}\text { Technique de } \\
\text { prélèvement }\end{array}$ & $\begin{array}{c}\text { MVS ( } \% \text { MES } \\
\text { totales) }\end{array}$ & $\begin{array}{c}\mathrm{COP} \text { (mg.kg } \mathrm{kg}^{-1} \\
\text { sec) }\end{array}$ & $\begin{array}{c}\text { Rendement } \\
\text { d'extraction en } \\
\text { chlorophylle a } \\
(\%)\end{array}$ & $\begin{array}{c}\text { CIP (mg.kg }{ }^{-1} \\
\text { sec) }\end{array}$ \\
\hline \multirow[t]{3}{*}{ «Amont " } & Trappe LABAM & $16,6 \pm 1,3$ & 3,1 & non mesurable & 6,5 \\
\hline & MIM & $17 \pm 4,5$ & 2,9 & non mesuré & 7,2 \\
\hline & $\begin{array}{l}\text { Prélèvement } \\
\text { manuel et } \\
\text { filtration }\end{array}$ & $21 \pm 2,1$ & non mesuré & sans objet & non mesuré \\
\hline \multirow[t]{4}{*}{ "Aval » } & Centrifugeuse & $10,9 \pm 0,1$ & 2,9 & $81 \pm 20$ & 7,4 \\
\hline & Trappe LABAM & $9,6 \pm 0,2$ & 3,1 & non mesurable & 9,35 \\
\hline & MIM & $10,2 \pm 9,8$ & non mesuré & $-6,4 \pm 30$ & non mesuré \\
\hline & $\begin{array}{l}\text { Prélèvement } \\
\text { manuel et } \\
\text { filtration }\end{array}$ & $23,5 \pm 1,4$ & non mesuré & sans objet & non mesuré \\
\hline
\end{tabular}


Les différences entre techniques de prélèvement sont moins marquées en juin 1995. Toutefois, les teneurs en MVS des échantillons prélevés par la trappe, le MIM ou la centrifugeuse sont nettement inférieures à celles issues du prélèvement manuel et des mesures sur filtres. Les teneurs en carbone inorganique particulaire, paramètre représentatif des carbonates particulaires présents dans l'échantillon, apparaissent analogues entre les diverses techniques de prélèvement.

Les rendements d'extraction de la matière phytoplanctonique, qui peut avoir un rôle important dans la fixation des métaux, par le MIM et la centrifugeuse font apparaître des écarts importants entre les deux techniques: la centrifugeuse collecte efficacement le phytoplancton, alors que le bilan moyen dans le MIM est nul et présente une forte variabilité dans le temps.

\subsection{Spéciation géochimique}

Les métaux présents dans un milieu aquatique peuvent s'associer à différents types de particules : argiles, hydroxydes de fer et de manganèse, précipités de carbonates, matière organique vivante ou détritique,... Selon le type d'association préférentielle, les métaux particulaires présenteront une écotoxicité potentielle variable : les interactions sont plus ou moins fortes selon le support particulaire considéré, et la remobilisation potentielle des métaux dépend donc de la nature de ce dernier. II importe donc de savoir si les diverses techniques d'échantillonnage permettent d'évaluer de manière comparable la répartition des métaux entre les diverses matrices particulaires.

A cette fin, des études de spéciation géochimique des micropolluants particulaires ont été mises en œuvre lors de l'expérience réalisée en juin 1995. Ces études consistent à remettre sélectivement en solution, par des procédures chimiques théoriquement spécifiques, les différents supports particulaires susceptibles d'avoir fixé une fraction de la pollution métallique. Ces supports sont, d'après la procédure de Tessier (Tessier et al., 1979) ici mise en œuvre, classés en 5 catégories:

1. Supports à sites échangeables : l'extraction consiste à remettre en solution les métaux fixés sur des sites non spécifiques des MES, par échange avec des cations tels que $\mathrm{Mg}^{2+}$.

2. Supports acido-solubles: l'extraction consiste à abaisser le $\mathrm{pH}$ afin d'attaquer les particules acido-solubles, telles que les précipités de carbonate.

3. Supports réductibles: en plaçant le milieu dans des conditions réductrices, on attaque des MES réductibles telles que les précipités d'oxydes de fer ou de manganèse.

4. Supports oxydables: l'attaque est dirigée vers les composés oxydables, et donc principalement, vers les matières organiques particulaires. 
5. Fractions résiduelles: par une attaque totale, analogue à celle qui est réalisée lors d'une minéralisation en une étape, on dissout les composés réfractaires aux précédentes extractions.

- Comparaison attaque totale-spéciation dans le cas du zinc: La somme des concentrations métalliques mesurées dans les différentes fractions extraites lors de la procédure de spéciation doit théoriquement être égale à la concentration mesurée par attaque totale unique. L'analyse des résultats obtenus pour le zinc permettent ainsi d'obtenir des informations intéressantes (tableau 9). En effet, les concentrations mesurées par minéralisation totale unique et par extractions successives apparaissent relativement proches, exception faite des concentrations déterminées sur l'échantillon prélevé par le MIM au point "Aval». Dans ce dernier cas, l'écart entre les concentrations mesurées par les deux méthodes atteint $37 \%$. La teneur en zinc de cet échan- tillon, déterminée par minéralisation totale unique, apparaissait, précisément, comme anormalement élevée par rapport à celles issues des échantillons prélevés par les autres méthodes (cf figure 5). Les résultats de spéciation confirment donc les doutes concernant la validité de la mesure de zinc particulaire total effectuée sur l'échantillon prélevé par le MIM au point "Aval " et fonderait l'hypothèse selon laquelle cet échantillon a été contaminé par une source externe de zinc.

- Répartition des métaux par fractions : La répartition des métaux entre les divers supports particulaires apparaît significativement différente selon les méthodes de prélèvement utilisées (figure 12). En particulier, l'observation la plus marquante est l'importance de la fraction dite "acido-soluble » dans l'échantillon prélevé par la centrifugeuse: alors que les métaux associés à cette fraction apparaissent négligeables, voire nuls, dans les échantillons prélevés par les

Tableau 9. - Comparaison des concentrations en zinc mesurées dans les échantillons de MES par minéralisation totale unique ou par extractions séquentielles

\begin{tabular}{|c|c|c|c|c|}
\hline Point & Technique & Attaque unique & $\begin{array}{l}\text { Somme des attaques } \\
\text {. successives }\end{array}$ & Ecart (\%) \\
\hline \multirow[t]{3}{*}{ «Amont $»$} & Trappe LABAM & $70 \pm 2$ & $67,9 \pm 8$ & -3 \\
\hline & Trappe LGA & $70,3 \pm 3,2$ & $70,5 \pm 8,2$ & $+0,3$ \\
\hline & MIM & $110,4 \pm 2,1$ & $116,6 \pm 6,4$ & $+5,3$ \\
\hline \multirow[t]{3}{*}{ “Aval» } & Trappe LABAM & $82,4 \pm 2,7$ & $93,1 \pm 5,3$ & +13 \\
\hline & MIM & $191 \pm 2,1$ & $121,1 \pm 13,7$ & $-36,6$ \\
\hline & Centrifugation & $87,6 \pm 1,6$ & $74,3 \pm 6,8$ & -15 \\
\hline
\end{tabular}



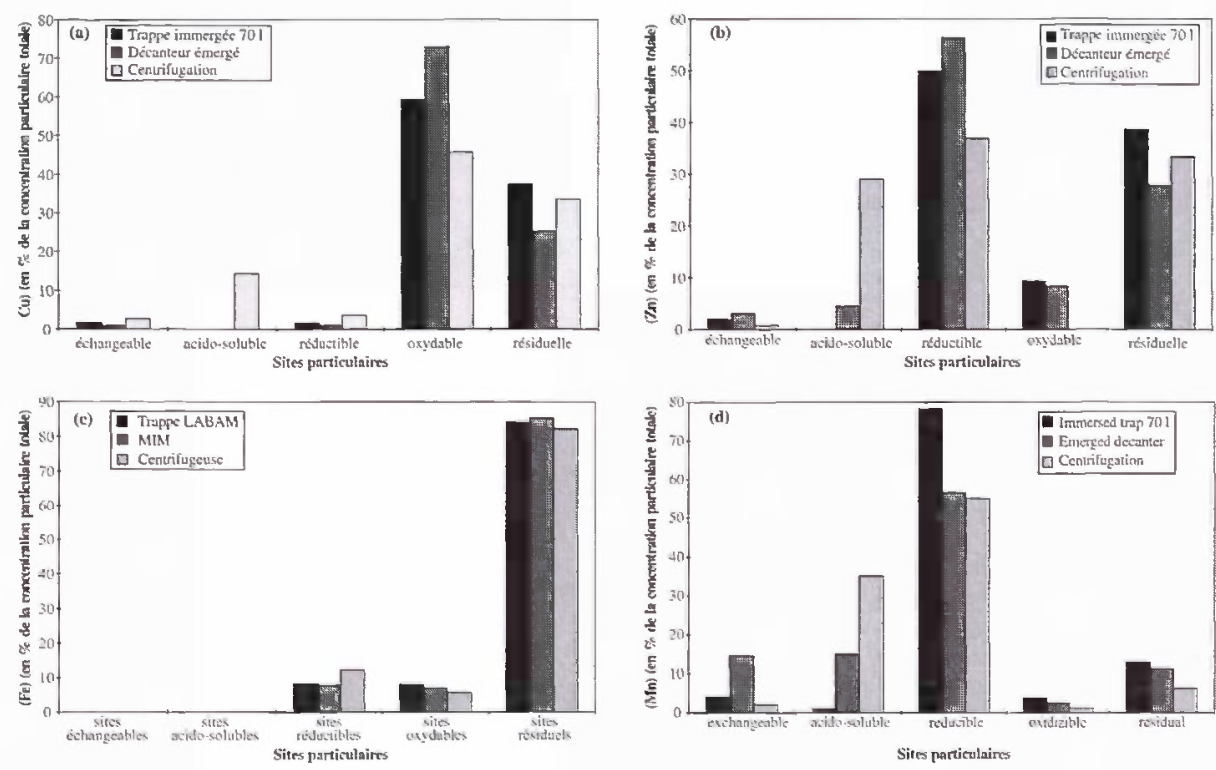

Fig. 12. - Répartition des métaux dans les différentes fractions particulaires - (a) : cuivre; (b) : zinc; (c) : fer; (d) : manganèse - Expérience de juin 1995 - Point "Aval "

Fig. 12. - Metals distribution among different particulate fractions - (a) : copper; (b) : zinc; (c) : iron; (d) : manganese - June 1995 - Station "Downstream »

trappes ou le MIM, leur contribution à la charge métallique totale de l'échantillon de centrifugation atteint respectivement 14,29 et $35 \%$ dans le cas du cuivre, du zinc et du manganèse. Ce point est fondamental, les analyses de spéciation devant aboutir à évaluer l'écotoxicité potentielle du sédiment; on considère en effet classiquement que les fractions dites "échangeable " et "acido-soluble " sont les plus facilement mobilisables et qu'elles présentent donc une écotoxicité potentielle plus élevée. Les valeurs reportées dans la figure 13 montrent que, selon la méthode de prélèvement employée, on peut aboutir à des conclusions fort différentes; par exemple, si l'on considère l'échantillon prélevé par centrifugation, on peut estimer que près d'un tiers du zinc est facilement remobilisable, alors que ce pourcentage tombe à environ $2 \%$ sur l'échantillon prélevé par la trappe LABAM. Plusieurs hypothèses peuvent être avancées pour expliquer ces différences:

$\rightarrow$ il est classique de considérer que la matrice particulaire préférentiellement remobilisée lors de l'attaque dite "acido-soluble" est essentiellement constituée par les précipités de carbonates. On pourrait donc envisager l'hypothèse selon laquelle la technique de centrifugation collecterait davantage de carbonates que les autres méthodes. Cette hypothèse est toutefois infirmée par les 


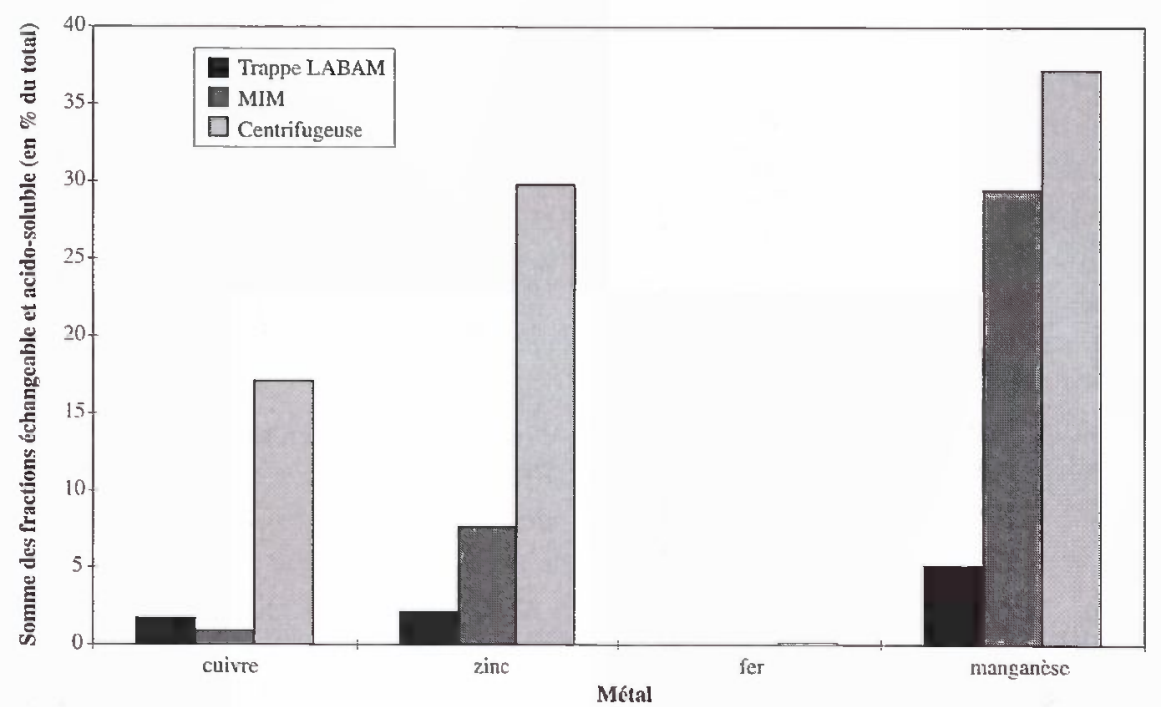

Fig. 13. - Somme des fractions échangeables et acido-soluble dans différents échantillons - Expérience de juin 1995 - Point "Aval"

Fig. 13. - Sum of exchangeable and acido-soluble fractions in different samples - June 1995 Station "Downstream »

mesures de CIP précédemment présentés (cf tableau 8)

$\rightarrow$ Meguellati (Meguellati, 1982) a montré que l'étape d'extraction dite «acido-soluble» remobilise non seulement les carbonates, mais qu'elle est également susceptible d'attaquer des composés organiques de petite taille; ainsi, il observe que près de $30 \%$ du cuivre associé à des acides humiques est remis en solution sous l'action des extractants utilisés pour l'attaque acido-soluble. Ces observations amènent à avancer l'hypothèse selon laquelle la centrifugeuse collecterait des colloïdes organiques dont une partie serait attaquée lors de l'extraction acido-soluble, colloïdes qui, compte-tenu de leur faible poids, ne seraient pas prélevés par les méthodes de décantation (MIM, trappes). $\rightarrow$ Meguellati (Meguellati, 1982) évoque l'influence des coatings organiques sur l'efficacité des extractions : il semble que la gangue organique qui se forme à la surface des particules minérales limite l'action des extractants chimiques, utilisés en particulier lors de l'attaque dite «acido-soluble". On peut ainsi avancer l'hypothèse seIon laquelle le stress mécanique provoqué par la centrifugation détruirait en partie ces coatings; l'attaque «acido-soluble » serait de ce fait facilitée. On peut également penser que les modifications granulométriques provoquées par la centrifugation entraînent une redistribution des métaux entre matrices particulaires.

L'analyse des résultats de spéciation permet par ailleurs de constater que les échantillons prélevés par le 
MIM sont plus riches en métaux associés aux matières oxydables. Bien que les mesures de COP précédemment présentées n'en fassent pas état, il semble donc que le MIM collecte plus de matières organiques que les autres méthodes, ou, du moins, que les matières organiques qu'il prélève présentent une plus grande affinité pour les métaux que celles extraites par les trappes ou la centrifugeuse.

\section{CONCLUSION}

Les MES constituant l'un des vecteurs majeurs de transport des micropolluants métalliques en rivière, il est essentiel de disposer de techniques de prélèvement permettant d'obtenir des échantillons représentatifs du milieu aquatique naturel. Pour pouvoir être qualifiée de "représentative", une technique de prélèvement doit répondre à divers critères : respect du seuil de coupure et du spectre granulométrique, respect de la nature des particules, emploi de matériaux inertes vis à vis des micropolluants devant être ultérieurement dosés. Plusieurs techniques de prélèvement (centrifugation, prélèvement séquentiel d'eau et filtration, filtration sur cartouches, trappes immergées, décanteur émergé (dit MIM)) ont été comparées afin de constater si elles satisfont l'ensemble de ces critères. Les principales conclusions issues de ces exercices d'intercomparaison sont les suivantes :

- Respect du seuil de coupure et du spectre granulométrique: L'analyse des spectres granulométriques de plusieurs échantillons (trappes, MIM, centrifugation) ont révélé de fortes différences, particulièrement lorsque les prélèvements sont réalisées en période de fort débit. Ainsi, les prélèvements par trappes apparaissent peu reproductibles en période de crue et privilégient la collecte de particules grossières. Par ailleurs, bien que conçus selon le même principe de prélèvement, en l'occurrence la sédimentation-décantation des particules, les trappes et le MIM collectent, en période de crue, des MES présentant des granulométries fortement différentes; en période d'étiage, les écarts sont moins marqués. Le spectre granulométrique de l'échantillon recueilli par la centrifugeuse montre quant à lui une forte contribution des particules les plus fines. Ces différences granulométriques induisent des écarts sur les concentrations métalliques totales mesurées dans les divers échantillons. Afin de réduire ces écarts, il est recommandé de normaliser les mesures brutes de métal particulaire total en considérant la proportion de particules appartenant à diverses classes granulométriques et les concentrations métalliques estimées pour chacune de ces classes. 
Cette normalisation est particulièrement utile pour le cuivre et le fer, qui présentent une affinité pour les particules fortement dépendante de la taille de ces dernières.

- Respect de la nature des particules: Les mesures permettant d'estimer la teneur en matière organique et inorganique de plusieurs échantillons (trappes, MIM, centrifugation) ont révélé des différences significatives en période de crue et de faibles écarts en période d'étiage. La spéciation biogéochimique des métaux particulaires semble dépendre quant à elle de la méthode de prélèvement utilisée. En particulier, l'échantillon recueilli par centrifugation présente une teneur en métaux facilement remobjlisable (fractions échangeable et acido-soluble) plus importante. Le choix de la méthode de prélèvement peut donc conduire à des conclusions différentes quant à l'écotoxicité potentielle des métaux particulaires.

- Emploi de matériaux inertes vis à vis des micropolluants devant être ultérieurement dosés : Les concentrations métalliques mesurées lors de la $1^{\text {ère }}$ campagne d'intercomparaison sur les échantillons recueillis par filtration apparaissent généralement supérieures à celles issues des autres méthodes d'échantillonnage. Ces résultats conduisent à supposer l'existence de contaminations lors des procédures de filtration, provoquées par la présence de métaux sur le filtre originel ou par l'adsorption d'une fraction des métaux dissous qui traversent le filtre lors du prélèvement. Par ailleurs, une contamination de l'échantillon du MIM par le zinc a été constatée lors de la $2^{\text {nde }}$ campagne d'intercomparaison.

Les résultats acquis fournissent des orientations pour améliorer les procédures de collecte des MES en rivière :

- L'emploi de filtres doit être soumis à des procédures de lavage et de contrôle préalables des filtres plus strictes.

- Le spectre granulométrique des échantillons collectés par les trappes doit être systématiquement mesuré afin de corriger les biais provoqués par les conditions locales d'écoulement. Des études ultérieures sont par ailleurs nécessaires afin de définir une procédure de normalisation granulométrique des concentrations métalliques mesurées dans les échantillons de trappes.

- Les procédures visant à définir la spéciation biogéochimique des métaux particulaires doivent impliquer des études complémentaires sur les méthodes de prélèvement des MES, et non pas seulement sur les schémas d'extractions proprement dits.

\section{RÉFÉRENCES}

Albrecht A., Reichert P., Beer J., Lück A., 1995. «Evaluation of the importance of reservoir sediments as sinks for reactor-derived redionuclides in riverine systems". Journal of Environmental Radioactivity, $V_{0}$ l 28, No3, p. 239-270. 
Battaglia A., Bonfanti G., Cattaneo C., De Pasquale N., 1984. "A method for field determinations of the chemical and physical characteristics of radionuclides after release into the river water ". In: $6^{\text {th }}$ Int. Congress of the International Radiation Protection Association, Vol.2, Verlag TUV Rehinland Gmbh, Köln, 891-894.

Ciffroy P., Vazelle D., Claveri B., 1994. "Méthode de mesure de micropolluants biodisponibles basée sur l'utilisation de bryophytes en milieu contrôle et sur un modèle d'interprétation quantitative: dossier technique ". Rapport EDF-DERHE31/94/14.

Ciffroy P., 1995. «Les échanges de radioélémnts à l'interface eau-matières en suspension. Principe théorique et équations d'un modèle descriptif. Bilan des expérimentations in situ ". Rapport EDF-DER HE31/95/21.

Ciffroy P., Siclet F., Humbert B., 1995. «In situ determination of the distribution of ${ }^{110 \mathrm{~m}} \mathrm{Ag},{ }^{58} \mathrm{Co},{ }^{60} \mathrm{Co}$ and ${ }^{54} \mathrm{Mn}$ between freshwater and suspended matter ". Proc. Int. Sym. on Environmental Impact of Radioactive Releases. IAEA. 812 May 1995.

Duinker J.C, Nolting R.F, van der Sloot H.A, 1979. "The determination of suspended metals in coastal waters by different sampling and processing techniques (filtration, centrifugation)". Netherlands Journal of Sea Research, 13 (2), 282-297.

Gardner W.D., 1980. "Field assessment of sediment trap : a laboratory evaluation". Journal of Marine Research, 38.

Leenaers $H_{\text {., }}$ 1989. "The transport of heavy metals during flood events in the polluted river Geul (The Netherlands)". Hydrological Processes, Vol. 3, 325-338.

Meguellati N., 1982. "Mise au point d'un schéma d'extractions sélectives des polluants métalliques associés aux diverses phases constitutives des sédiments". Thèse de I'Université de Pau et des Pays de l'Adour.

Taconnet J., 1995. «Impact d'un rejet industriel sur les concentrations métalliques particulaires en Seine". Rapport de stage, Maitrise Sciences et Techniques Génie sanitaire et environnement, Université Paris XII, Val de Marne

Tessier A., Campbell P.G.C., Bisson M., 1979. "Sequential extraction procedure for the speciation of particulate trace metals $\%$. Analyt. Chem., 51, $p$. 844-851. 